\title{
Feasibility Study of a Partially Released Lead-Zirconate-Titanate (PZT) Diaphragm Micro-actuator
}

\author{
Yifeng Liu*, ChuanLuo**, G. Z. Cao ${ }^{\dagger}$, I.Y Shen
}




\begin{abstract}
Piezoelectric thin-film micro-sensors and actuators often appear in the form of a diaphragm anchored around its entire boundary. When a micro-device is scaled down in size, its sensitivity reduces and natural frequency increases, drastically lowering its performance. The purpose of the paper is to study the feasibility of introducing through-etched slots to partially release the diaphragm at its anchor. As a result, a micro-device can be scaled down in size without significantly altering its sensitivity and natural frequency. In this paper, we first present a finite element simulation proving the concept. We then describe processing steps to fabricate a leadzirconate-titanate (PZT) thin-film diaphragm sensor/actuator with a partially released boundary. Challenges encountered in the fabrications, such as cat ear and electrode non-uniformity, are explained and overcome. As a case study, we demonstrate the feasibility to design, fabricate, and test an intra-cochlear micro-actuator probe that employs three partially released PZT diaphragms at the tip of a cantilever. Experimental measurements indicate that the sensitivity is dominated by the diaphragm deflection, while the first natural frequency is dominated by the cantilever structure. Finite element simulations not only confirm the experimental measurements but also optimize diaphragm dimensions for sensor/actuator performance.
\end{abstract}




\section{INTRODUCTION}

Piezoelectric thin-film micro-devices have found wide applications, such as pressure sensors [1][2], acoustic actuators [3][4][5], bio-sensors [6][7], bio-actuators [8], micro-pumps [9][10], microphones $[11][12][13][14][15][16][17]$, micro power generators $[18][19]$, and energy harvesting transducers [20]. A common design of such devices is a diaphragm structure; see Figure 1. Usually, the design consists of a diaphragm, electrodes, and diaphragm anchors. The diaphragm is often composite, with a piezoelectric (e.g., lead-zirconate-titanate (PZT) or aluminum nitride (AlN)) thin film over a base material (e.g., silicon, silicon oxide, silicon nitride, or combination thereof). Electrodes can appear above or below the piezoelectric thin film or both. The electrodes can be made from gold or platinum with an adhesion layer (e.g., chromium or titanium). The anchor, formed by bulk materials surrounding the diaphragm, provides a fixed boundary condition to support the diaphragm. When voltage is applied to the piezoelectric thin film, it extends or contracts flexing the diaphragm serving as an actuator. Conversely, when the diaphragm deforms, the piezoelectric thin film produces electric charges serving as a sensor. 


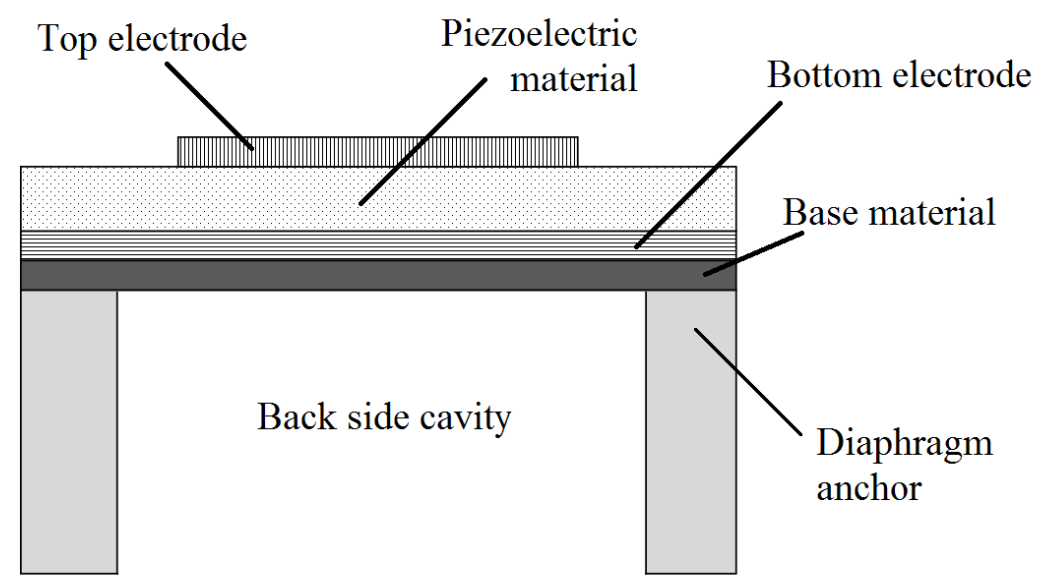

Figure 1.Cross sectional view of a typical piezoelectric sensor or actuator with a diaphragm structure (not in scale)

Performance of piezoelectric micro-sensors and actuators is often evaluated in terms of two parameters: sensitivity and frequency bandwidth. For piezoelectric thin-film micro-sensors, the sensitivity is usually the charge generated per unit force or displacement (e.g., $\mathrm{pC} / \mathrm{Pa}$ or $\mathrm{pC} / \mathrm{nm}$ ). For piezoelectric thin-film micro-actuators, the sensitivity is usually the displacement or force delivered per applied voltage (e.g., $\mathrm{nm} / \mathrm{V}$ or $\mathrm{N} / \mathrm{V}$ ). The frequency bandwidth is the frequency range in which frequency response functions of the micro-sensors or actuators remain roughly constant. For example, design of an intra-cochlear micro-actuator as a hearing aid aims at displacement of $200 \mathrm{~nm}$ over the audible range [3]. Given a 5V power source, the intra-cochlear micro-actuator would have a sensitivity of $40 \mathrm{~nm} / \mathrm{V}$ with a frequency bandwidth from $10 \mathrm{~Hz}$ to $12 \mathrm{kHz}$. 
When a micro-device is scaled down proportionally in size, physical laws mandate that the device's natural frequency and stiffness increase accordingly. The increase in the natural frequency results in a surplus of the frequency bandwidth, while the increase in the stiffness often causes a significant drop of the sensitivity. Therefore, one major challenge encountered in the design of diaphragm-type piezoelectric micro-devices is to maintain the desired sensitivity and frequency bandwidth while reducing the size of the devices.

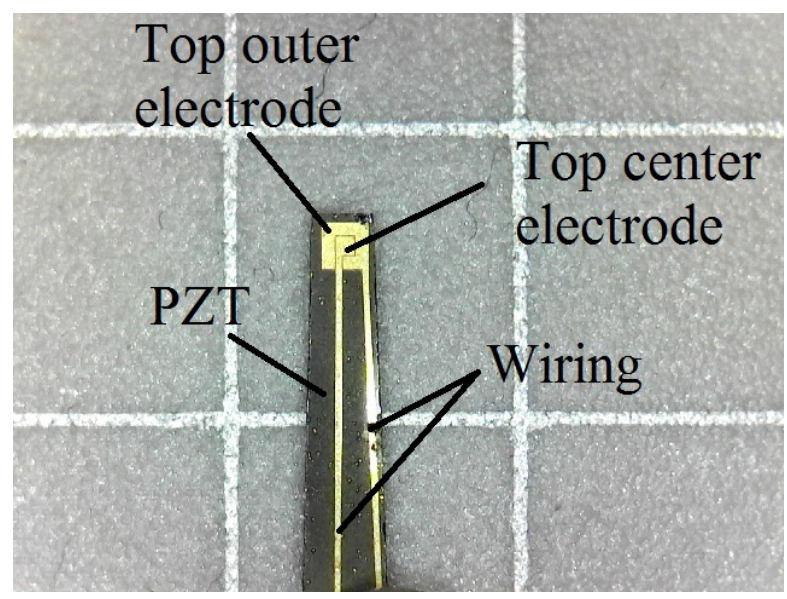

(a) Front side of the first generation actuator

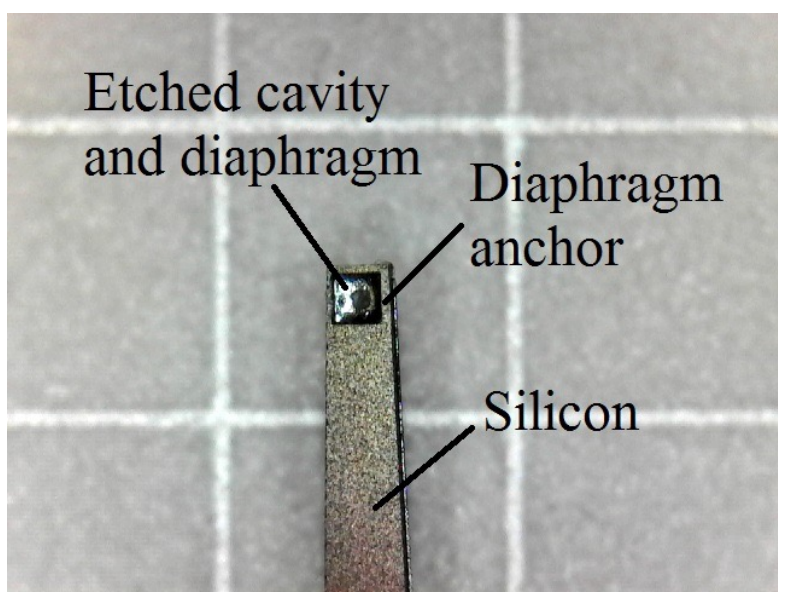

(b) Back side of the first generation actuator

Figure 2. Front and back views of the first-generation PZT micro-actuator probe

As an example, a PZT thin-film acoustic micro-actuator was developed as a hearing aid to be placed in cochlea [3]. The goal is to generate acoustic waves directly in cochlea and to coordinate with a traditional cochlear implant that generates electric stimulation to enhance speech recognition. Figure 2 shows the top and bottom views of a first-generation (1-G) intra- 
cochlear probe that carries an acoustic micro-actuator. The 1-G probe is in the form of a cantilever with a cross-sectional area of $0.4 \mathrm{~mm} 1 \mathrm{~mm}$. The acoustic micro-actuator is located at the tip of the probe in the form of a diaphragm (cf. Figure 1). Moreover, the diaphragm is 0.8 $\mathrm{mm} 0.8 \mathrm{~mm}$ in size and is $2-3 \mu \mathrm{m}$ in thickness. The diaphragm is anchored all around at four sides. The diaphragm and the anchors are formed simultaneously through a deep reactive ion etch (DRIE) from the bottom side of the probe. A finite element analysis (see Appendix 1) indicates that the sensitivity is $18.22 \mathrm{~nm} / \mathrm{V}$ and the first natural frequency is $35.51 \mathrm{kHz}$. The $1-\mathrm{G}$ intra-cochlear, acoustic micro-actuator probe has gone through several rounds of animal tests and proved to be successful [21]. Also, the 1-G probe was made in the form of a cantilever for acute in-vivo tests, where ease of implantation was important and the test duration was short. For chronic in-vivo tests or realistic hearing devices, an intra-cochlear microactuator would only include the diaphragm portion with a small wiring area. The total length may not exceed $1 \mathrm{~mm}$.

Despite the success, the 1-G intra-cochlear micro-actuator probe is still a bit too large; it barely enters the basal turn of cochlea. A more desirable intra-cochlear micro-actuator probe is $30 \%$ or even $50 \%$ smaller in size. The smaller size will allow surgeons to place the acoustic micro-actuator at an optimal location (e.g., facing the basilar membrane where hair cells reside) with proper bone anchor. The size reduction, however, will undermine the sensitivity and bandwidth. Based on the analysis in Appendix 1, if the 1-G intra-cochlear micro-actuator probe is reduced $50 \%$ in size with proportion, the sensitivity will be reduced to $3.39 \mathrm{~nm} / \mathrm{V}$ while the first natural frequency will be increased to $231.28 \mathrm{kHz}$. The sensitivity is significantly smaller, and the frequency bandwidth is substantially bigger than the needed audible frequency range. In 
this case, what would be an alternative design that could provide a smaller size without significantly altering the sensitivity and natural frequency?

A natural solution is to adopt a cantilever diaphragm to substantially reduce the stiffness [22] [23]. Use of a cantilever diaphragm, however, has tradeoffs. First, there are practical considerations in reliability. For example, cantilever diaphragms tend to warp more than fully anchored ones when residual stresses are present. Cantilever diaphragms are more fragile because they are only supported at one end. Without any protection, cantilever diaphragms could break during the implantation process of a surgery. Second, cantilever diaphragms do have their own design challenges. As an acoustic actuator, a fully anchored diaphragm behaves like a speaker displacing its ambient fluid effectively. It is not clear if a cantilever diaphragm will be as effective. Another design constraint is the thickness of the PZT thin film. Under existing technologies, PZT thin films with thickness ranging from several to tenths of micrometers are possible but challenging to fabricate for cantilever structures. As a result, choice of thickness and bandwidth for cantilever diaphragms may not be unlimited. It would be very desirable to have an alternative design to scale down a diaphragm actuator without significantly altering the sensitivity and natural frequency.

Motivated by the considerations above, we propose an alternative design by modifying the boundary conditions of the fully anchored diaphragm at the anchors. For example, one strategy is to partially release the anchors on two opposite sides of the diaphragm; see Figure 3. The presence of the free boundaries softens the micro-devices, keeping the sensitivity and natural frequency in check. Also, the amount and location of the released boundary can be adjusted to 
achieve a desired sensitivity and natural frequency. This alternative design has not been pursued actively in the research community and its potential remains largely unexplored.

The purpose of this paper is to study the feasibility of using a partially released diaphragm design for micro-devices. In particular, we implement the partially released boundaries by using through-etched slots. The study includes design of the partially released boundaries, fabrication of the devices, experimental characterization of the devices, and performance optimization. This study is neither trivial nor incremental, because there are several critical issues that need to be addressed.

First, the design of the partially released boundaries is complicated by the presence of residual silicon. For example, the diaphragm of the 1-G micro-actuator probe shown in Figure 2 was formed via a DRIE from the backside of the silicon wafer. Since the etch rate is not uniform, there is always some un-etched silicon in the form of residue building up at the diaphragm-anchor boundary (cf. Figure 2 of [26] or Figure 16(b) of Appendix 1). When the size of the micro-devices is reduced, the residual silicon does not decrease its size accordingly. As a result, the residual silicon could detrimentally affect the sensitivity and the natural frequency. Consequently, the location and size of the etch slots will largely determine the size of the residual silicon and thus the final performance, even after the partially released boundaries are formed.

Next, new fabrication processes must be developed to accommodate the partially released boundary while making the micro-devices. The use of through-etched slots to create partially released boundary implies that six different layers (i.e., PZT, top electrode $(\mathrm{Au} / \mathrm{Cr})$, bottom electrode (Pt/Ti), silicon oxide, silicon nitride, and silicon) need to be separately patterned and 
etched. Although patterning and etching formulas of individual layers are available [27][28], combined use of the formulas to implement such etch slots is not a trivial task. For example, the multi-layer patterning requires very accurate alignment. Moreover, any subsequent processing over a patterned substrate surface may introduce various problems, such as layer delamination, "cat ear" created by lift-off, and non-uniformity in etching.

Finally, design issues may appear when a partially released diaphragm is incorporated in a micro-device. For example, how would a host structure of a micro-device affect the performance of a partially released diaphragm? What happens when multiple diaphragms appear in a microdevice?

For the rest of the paper, we will present how we resolve these three issues. We first present a design of a PZT thin-film micro-actuator with reduced dimensions and partially released boundary conditions. A finite element simulation is conducted to prove the concept. Then we explain the fabrication processes and discuss in detail some unique steps that enable the fabrication. We then demonstrate the feasibility to fabricate the new design on an intra-cochlear acoustic micro-actuator probe that employs three partially released diaphragms. Performance of the micro-actuator probe (e.g., sensitivity and natural frequency) is then measured using a laser Doppler vibrometer (LDV). Finally, we conduct a finite element simulation to confirm the measurements and optimize the diaphragm dimensions. 
(a) top view of the diaphragm

partially released actuator model (b) bottom view of the diaphragm

partially released actuator model

Figure 3. A diaphragm partially released PZT micro-actuator model:

(a) front side view, (b) back side view

\section{DESIGN OF PARTIALLY RELEASED DIAPHRAGM MICRO-ACTUATORS}

The scale-down finite element model in Appendix 1 is revisited here. To partially release the anchors, we introduced two through-etched slots at two opposite sides of the diaphragms; see Figure 3(a). Note that the through-etched slots also partially remove the residual silicon that hinders the diaphragm from deforming, as seen in Figure 3(b). Note that the diaphragm is now rectangular (i.e., $550 \mu \mathrm{m} 340 \mu \mathrm{m}$ ). That the long sides are free further reduces the stiffness of the diaphragm. Also, there is one central electrode and two side electrodes. The central and side electrodes can be driven in an out-of-phase manner to enhance the actuator displacement.

A static analysis and a modal analysis are conducted for this model. The static deflection of the diaphragm is $12.62 \mathrm{~nm} / \mathrm{V}$ and the first natural frequency is $86.64 \mathrm{kHz}$. The partially released boundary significantly improves the performance of the fully anchored scaled-down model (cf. $3.39 \mathrm{~nm} / \mathrm{V}$ and $231.28 \mathrm{kHz}$ ). Moreover, the performance of the partially released design is similar to that of the fully anchored full-scale model (cf. $18.22 \mathrm{~nm} / \mathrm{V}$ and $35.51 \mathrm{kHz}$ shown in Appendix 1). Note that the dimensions chosen in Figure 3 are not optimized yet. As will be seen 
later, the optimized model can reach a sensitivity of $29.59 \mathrm{~nm} / \mathrm{V}$ and a first natural frequency of $32.24 \mathrm{kHz}$, which are equivalent to those of a fully anchored full-scale micro-actuator model.

\section{FABRICATION OF PARTIALLY RELEASED MICRO-ACTUATORS}

A critical step to fabricate the through-etched slots is to align and pattern each layer separately. Figure 4 shows fabrication steps of a partially released, PZT thin-film, diaphragm micro-actuator via multi-layer patterning.

First of all, all samples start with standard $100-\mathrm{mm},<100>$ silicon wafers as substrates. After proper wafer cleaning, $5000 \AA$ silicon oxide was grown on both sides of the substrate via wet thermal oxidation. $2000 \AA$ of silicon nitride was then deposited through low-pressure chemical vapor deposition (LPCVD); see Figure 4(a). The first alignment pattern was formed by dry-etching the silicon nitride layer, exposing the underneath silicon oxide. The first alignment pattern serves as a reference, and each subsequent layer was aligned accordingly to avoid accumulation of patterning errors.

Next, titanium and platinum were e-beam deposited and patterned by lift-off as the bottom electrode. The samples were then annealed to relieve internal stresses (Figure 4(b)). The PZT thin film was fabricated via a sol gel method. Specifically, PZT sol was first made and spincoated onto the sample surface. The samples were then quickly transferred to furnace and sintered at $650^{\circ} \mathrm{C}$ to form perovskite structured PZT crystal [29]. After repeating the spin coating and the sintering for 3 times, a PZT layer of 0.8-0.9 $\mu \mathrm{m}$ was formed. Afterwards, the PZT was 
patterned via wet etching using 10:1 BHF bath for 15 minutes and $31.45 \% \mathrm{HCl}$ bath for 5 minutes [28] (Figure 4(c)). Chromium and gold were then e-beam deposited and patterned by lift-off as top electrodes (Figure 4(d)).

Finally, double side etching was employed to fabricate the through-etched slots and to release the actuator probes simultaneously. On the topside, the slot areas and the actuator separation lines were first etched using deep reactive ion etching (DRIE) for approximately 100 $\mu \mathrm{m}$ (Figure 4(e)). On the bottom side, DRIE was performed over the entire diaphragm areas and the separation line areas till the silicon oxide is exposed (Figure 4(f)). At this point, the actuators shown in the dotted rectangle of Figure 4(f) were automatically separated from the substrate, ready for wiring, soldering and testing. 


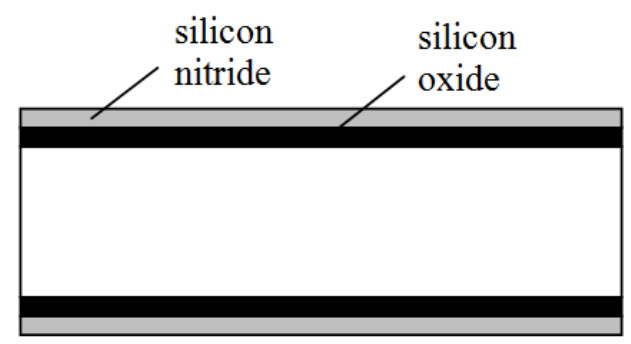

(a) Insulation layer: silicon oxide and silicon nitride

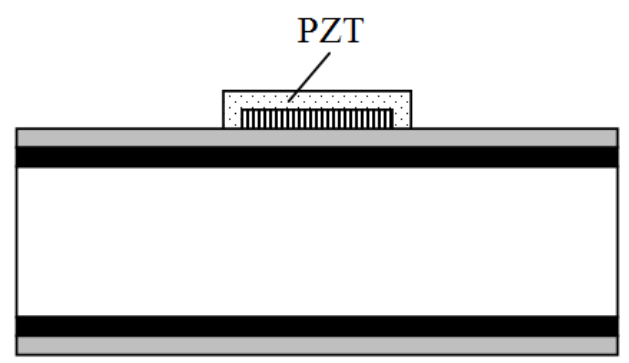

(c) PZT deposition, sintering and patterning

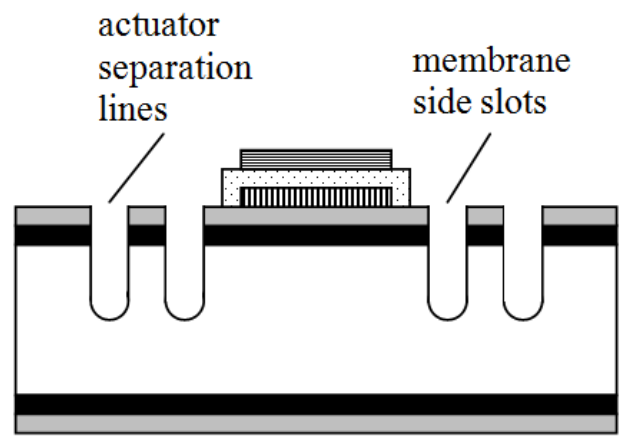

(e) Front side etching bottom electrode

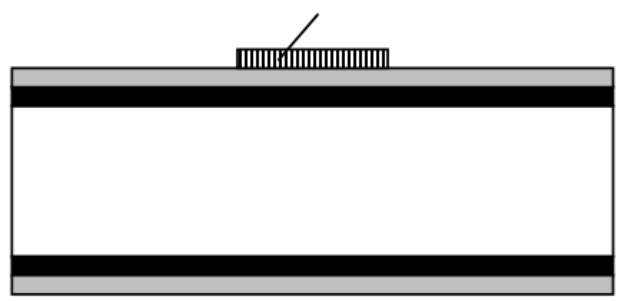

(b) Bottom electrode deposition patterning and annealing

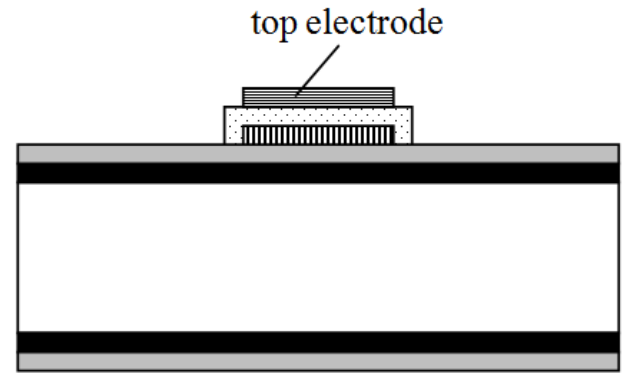

(d) Top electrode deposition and patterning

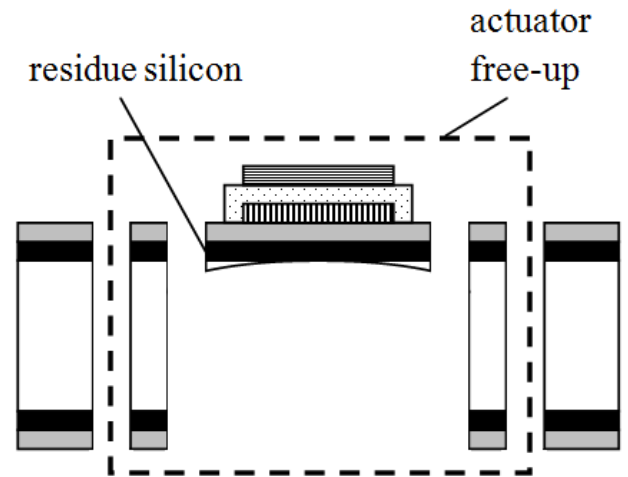

(f) Back side etching, membrane formation and actuator free-up

Figure 4. Fabrication flow of the diaphragm partially released PZT micro-actuator 
For a successful fabrication run, two issues require special attention: (a) elimination of cat ear and (b) annealing of the bottom electrodes. They are explained in detail as follows.

\subsection{Elimination of Cat Ear}

It is commonly accepted that negative photo-resist produces more accurate patterns in a lift-off process. We, however, use positive photo-resist (AZ1512 from AZ Electronic Materials) for the lithographic patterning of the bottom electrode for the following reasons. First, the pattern of the bottom electrode is relatively simple, and a high accuracy is not required. Second, positive photo-resists have much better adhesion property to the silicon nitride layer than the negative photo-resists. This is especially important, because the substrate experiences high temperature (about $250^{\circ} \mathrm{C}$ ) and thus large thermal expansion during e-beam deposition of platinum. Our fabricated results repeatedly showed that negative photo-resist peeled off and did not survive the e-beam deposition.

The use of the positive photo-resist, however, result in "cat ear," which is long, tall residual platinum formed during the lift-off. Figure 5(a) shows the surface profile of the substrate with the bottom electrode, and each division in Figure 5(a) is $1.1 \mu \mathrm{m}$. The central portion is the bottom electrode, and the outer portions are the substrate. The two sharp peaks with a height of $9 \mu \mathrm{m}$ are cat ear. The thick cat ear, if not eliminated, will penetrate the PZT layer and connect with the top electrode causing a short circuit. 
(a) thickness profile of the substrate and the bottom electrode surface immediately after the lift-off; the height of the cat ear is $9 \mu \mathrm{m}$

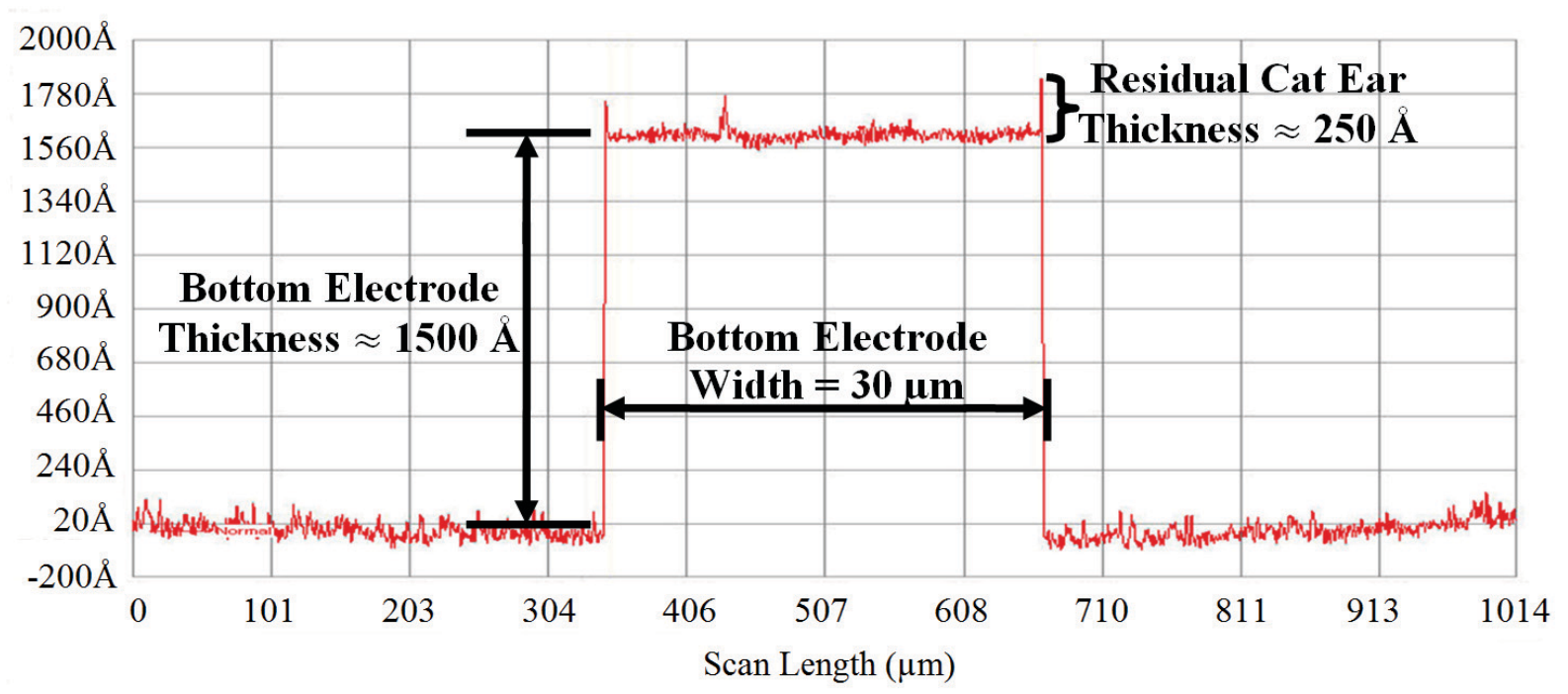

(b) thickness profile of the substrate and the bottom electrode surface after sonicating bath and before annealing; the height of the cat ear is reduced to $250 \AA$

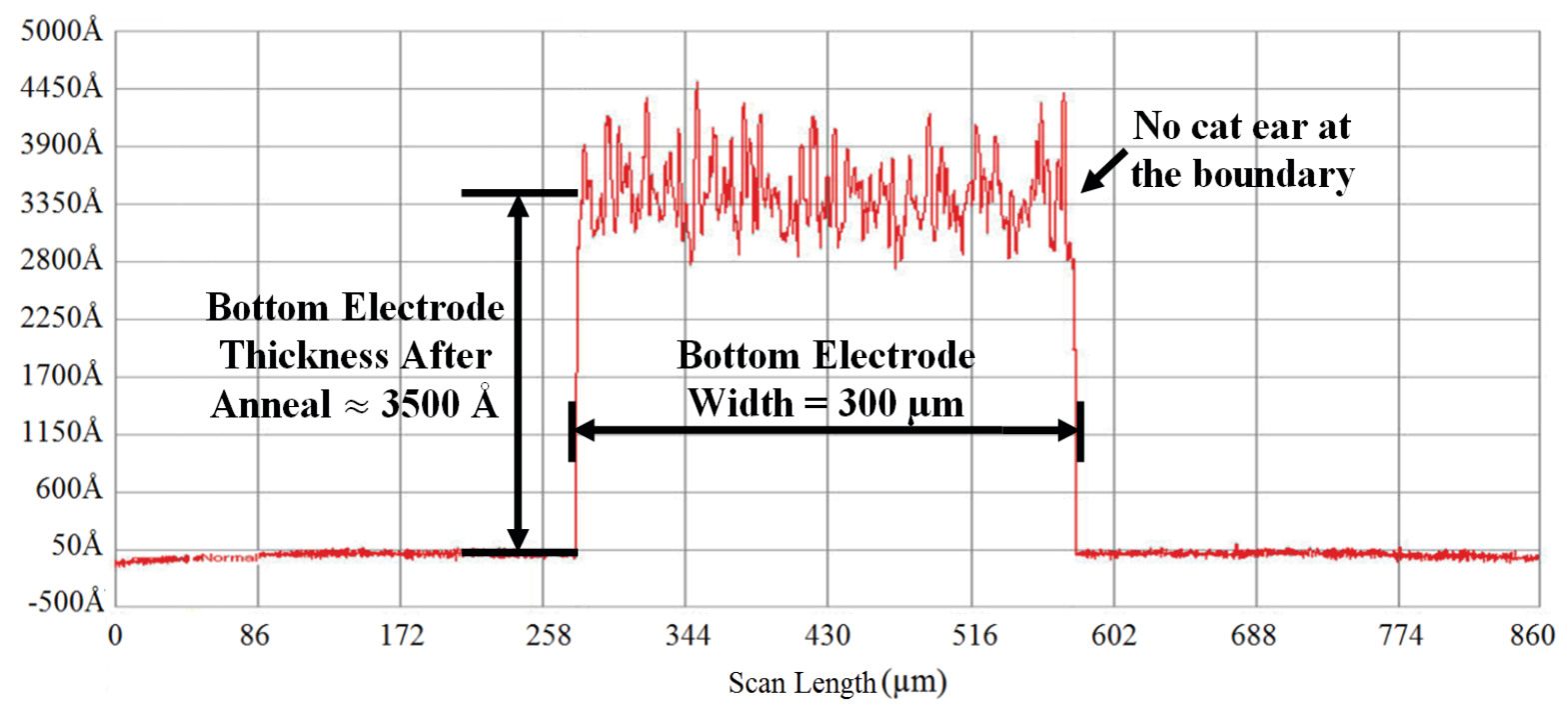

(c) thickness profile of the substrate and the bottom electrode surface after annealing; no cat ear at the boundary

Figure 5. The thickness profile of the bottom electrode tip and the substrate surface:

(a) after the lift-off, (b) after sonicating bath and before annealing, and (c) after annealing. 
The long and narrow cat ear was physically broken via a sonicating bath. After the sonicating bath, only short residue of the cat ear (about $250 \AA$ ) was left at the pattern boundaries; see Figure 5(b). Furthermore, the residue was totally eliminated after the bottom electrode was annealed; see Figure 5(c).

\subsection{Bottom Electrode Annealing}

The bottom electrode must be annealed to release internal stresses accumulated during its deposition process. With proper annealing conditions (e.g., [30]), the resulting bottom electrode shows a highly porous structure (Figure 6(a)) to anchor the PZT thin film [30]. Moreover, past experience indicates that $30-35 \%$ porosity (i.e. void area vs. total area) leads to the best PZT deposition (Figure 6(a)). Less porous (under-annealed) and more porous (over-annealed) conditions will cause PZT delamination or cracking. One recipe for annealing the bottom electrodes is to ramp from room temperature to $800^{\circ} \mathrm{C}$ in 60 minutes, dwell for $15-25$ minutes to control the porosity, and to cool down naturally in furnace.

When the bottom electrode is etched into patterns and annealed, a new challenge emerges. The porosity is non-uniformly distributed throughout the annealed bottom electrode. If the central area is properly annealed, the electrode near the boundaries (typically $60-100 \mu \mathrm{m}$ into the electrode) becomes under-annealed; see Figure 6(b). After the PZT thin film is deposited, the film starts to crack and delaminate at the boundaries.

We hypothesize that the non-uniform distribution of porosity results from two factors. First, there is still some bottom electrode "cat ear" at the pattern boundaries before annealing (Figure 5(b)). The metal consisting the "cat ear" will eventually migrate and merge into the 
bottom electrode near its boundaries, causing those areas slightly thicker than central area. Based on the law of diffusion, the thicker areas take longer time to complete the annealing than the thinner areas. Second, the surface topology of the substrate is not uniform. The platinum bottom electrode is deposited over some areas while the silicon nitride is exposed at other areas. Such non-uniformity in surface topology will also cause the annealing to become non-uniform.

The above two factors (i.e., non-uniformity for bottom electrode thickness and surface topology) will have significant influence on annealing when temperature changes rapidly (such as in the short time dwelling and the fast cooling down). Theoretically, if enough dwelling time is given, or the temperature changes slowly, the annealing will become much more uniform. In that case, only the dwelling temperature determines the final porosity state. Based on the reasoning above, we implement a new annealing strategy to minimize the non-uniform porosity distribution. The new strategy dwells at $790^{\circ} \mathrm{C}$ for 60 minutes to ensure a fully annealed condition. To prevent fast temperature drop, the furnace is not turned off after dwelling. Instead, a linear and slowly declined (more than 9 hours) temperature profile is programmed to control the temperature of the furnace. Figure 6(c) shows the porous structure of the bottom electrode near the patterning boundaries. The porosity is significantly improved and becomes properly annealed (cf. Figure 6(b)). 


\begin{tabular}{|c|c|c|}
\hline 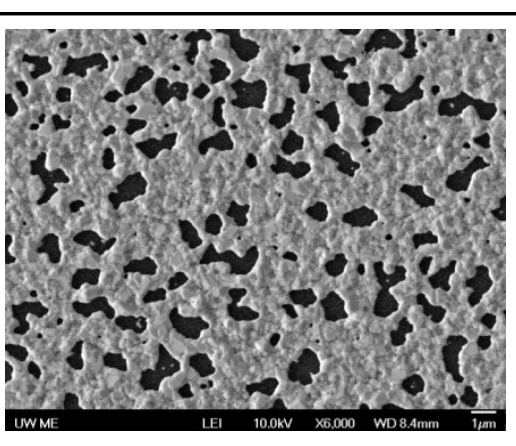 & 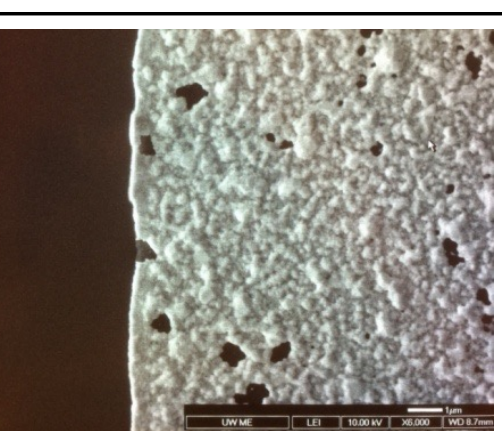 & 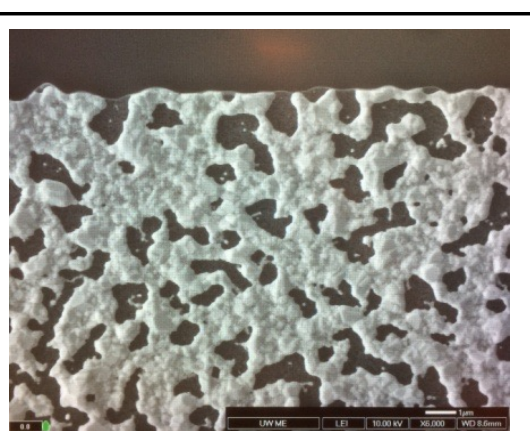 \\
\hline $\begin{array}{l}\text { (a) Properly annealed at the } \\
\text { center under the old scheme } \\
\text { [30] }\end{array}$ & $\begin{array}{l}\text { (b) under-annealed near the } \\
\text { boundary under the old } \\
\text { scheme [30] }\end{array}$ & $\begin{array}{l}\text { (c) properly annealed near the } \\
\text { boundary with the new } \\
\text { scheme }\end{array}$ \\
\hline
\end{tabular}

Figure 6. The bottom electrode annealing: (a) properly annealed at the center under the old scheme [30]; (b) under-annealed near the boundary under the old scheme [30]; (c) properly annealed near the boundary with the new scheme.

\section{CASE STUDY: AN INTRA-COCHLEAR MICRO-ACTUATOR PROBE}

In this section, we will demonstrate how the partially released diaphragm (cf. Figure 3) can be used to reduce the size of $1-\mathrm{G}$ intra-cochlear micro-actuator probe in Figure 2. The demonstration includes (a) layout design and fabrication, (b) experimental setup, (c) measured results, and (d) discussions. They are explained in detail as follows.

\subsection{Layout Design and Fabrication}

The second-generation (2-G) intra-cochlear micro-actuator probe still adopts a cantilever structure, but it hosts three partially released diaphragms; see Figure 7. Each diaphragm has a size of $550 \mu \mathrm{m} 340 \mu \mathrm{m}$, i.e., same as Figure 3(a). There are two designs. For the first design shown in Figure 7(a), the three diaphragms are synchronously driven through a central electrode and a side electrode. This design aims to displace maximal amount of fluid in cochlea achieving 
better hearing rehabilitation. For the second design shown in Figure 7(b), the three diaphragms are independently driven via individual electrodes. As a result, some diaphragms can serve as actuators and others as sensors. This design aims to deliver a smart probe that can sense and actuate simultaneously. In both designs, the diaphragms' layout follows the analysis in Figure 3 and the fabrication follows the step shown in Figure 4. The only difference in these two designs is the layout of the electrodes.

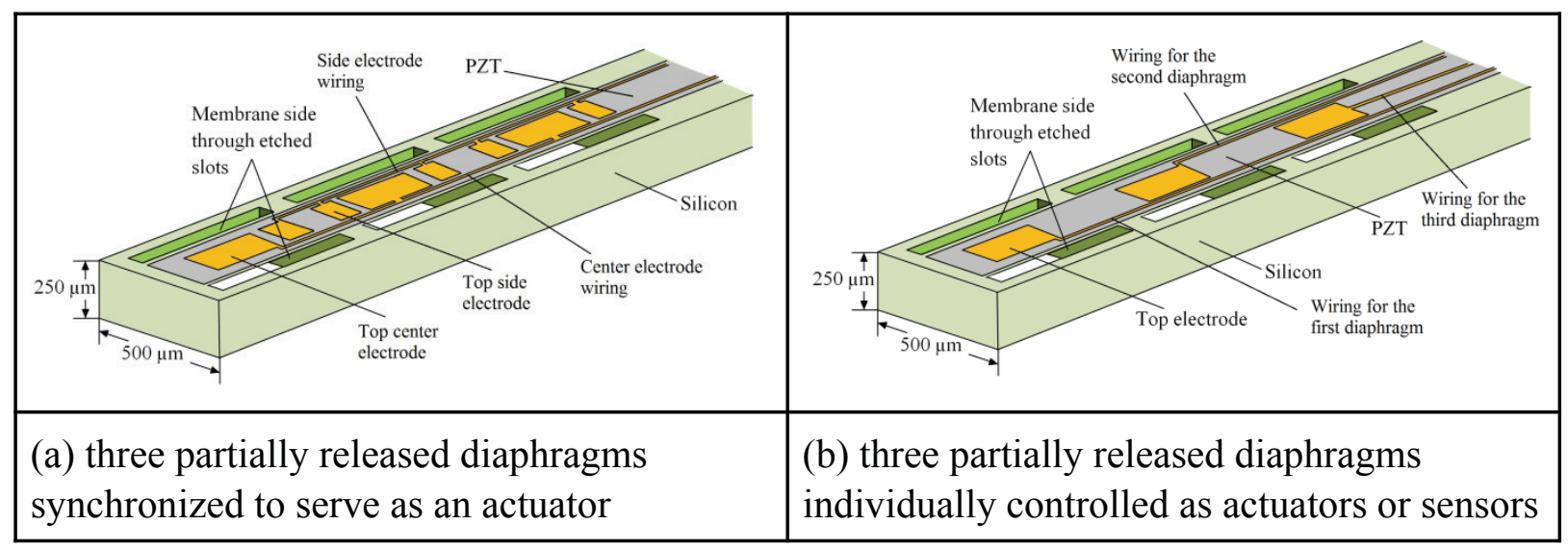

Figure 7. Layout design of 2-G micro-actuator probes: (a) three partially released diaphragms synchronized to serve as an actuator, and (b) three partially released diaphragms individually controlled as actuators or sensors

The cantilever structure of the $2-\mathrm{G}$ probe is intended for acute in-vivo tests, because the cantilever form makes it easy for surgeons to implant the probe into cochlea. Since the animal is sacrificed at the end of the test, the animal is in anesthesia during the entire test. There is no risk of breaking the probe even the cantilever sticks out of the animal body. For chronic in-vivo tests or viable hearing devices, the microactuator must be completely implanted. In that case, the intra-cochlear microactuator would only include the diaphragm portion with a small wiring area. 
Figure 8 shows a photo of the first design (cf. Figure 7(a)) fabricated via the steps shown in Figure 4. There are three diaphragms, and each has two, 30- $\mu \mathrm{m}$ wide, through-etched slots on the side serving as free boundaries. Each diaphragm has a center electrode and one or two side electrodes. The three center electrodes are connected in parallel. The side electrodes are close to the fixed anchors of the diaphragms. All the side electrodes are also in a parallel connection. When the center and side electrodes are driven in an out of phase manner, the diaphragms will produce the maximal displacement ${ }^{1}$ [4]. Figure 8 also shows a string of human hair for comparison.

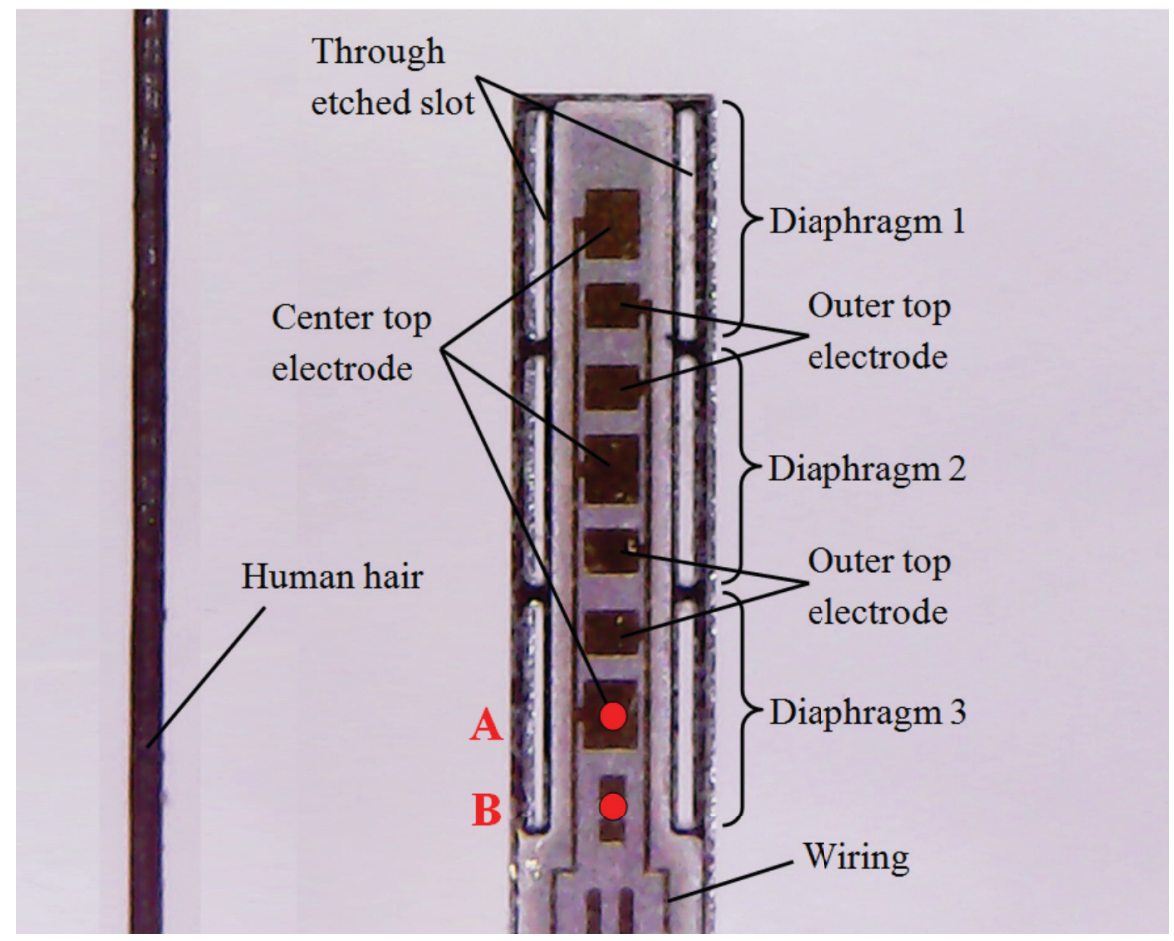

Figure 8. Optical microscope view of the fabricated partially released actuator

\footnotetext{
${ }^{1}$ When the diaphragm vibrates, the central portion and the side portions near the anchor have opposite curvatures (i.e., one concave upwards and the other concave downwards). In other words, when the center portion experiences elongation, the side portions experiences compression, and vice versa. Therefore, the center and the side electrodes need to be driven out of phase in order to produce the maximal displacement.
} 
compared with a string of human hair

\subsection{Experimental Setup}

The 2-G micro-actuator probe in Figure 8 is then cantilevered to a piece of slide glass, which is, in turns, glued to a heavy aluminum block; see Figure 9(a). Both attachments employ epoxy, which is stiff enough to be considered as "fixed boundary conditions" [27].

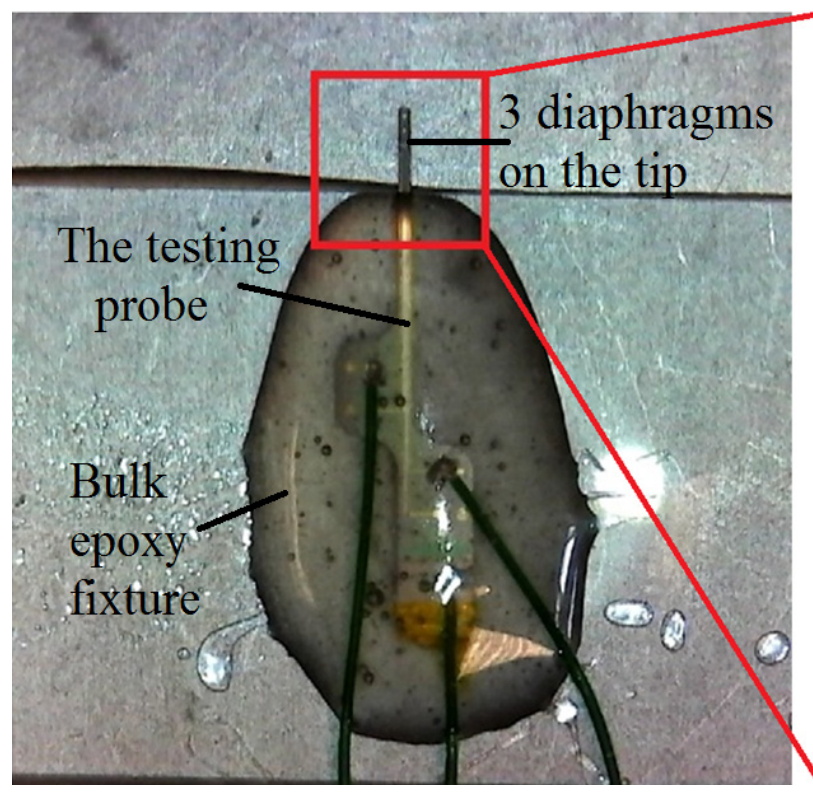

(a) overall configuration of the actuator fixture

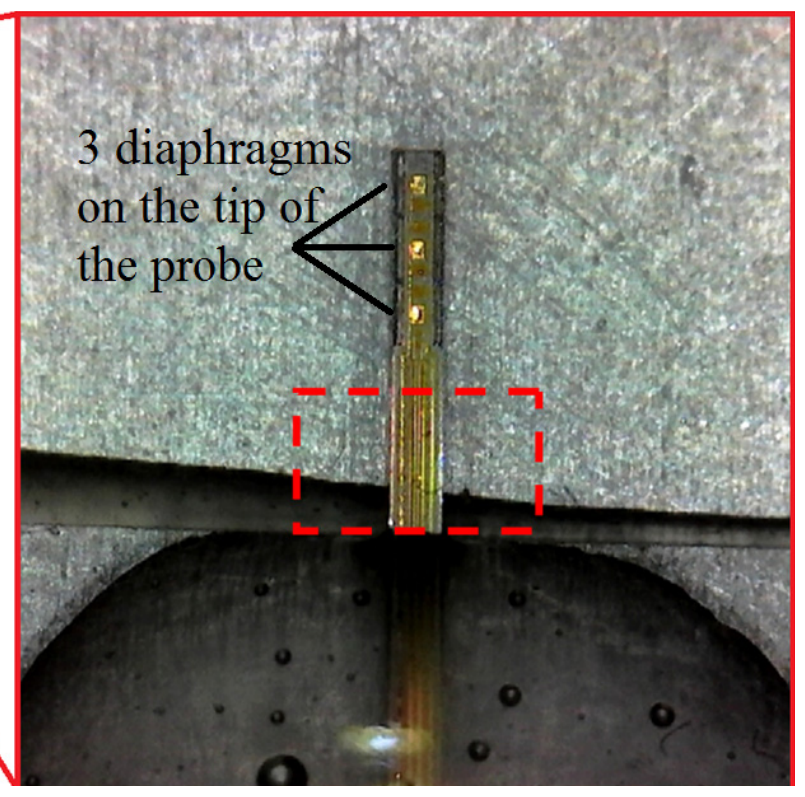

(b) blow-up view of the tip configuration

Figure 9. Experimental setup of the 2-G intra-cochlear micro-actuator probe:

(a) fixture via epoxy, (b) blow-up view 
The setup characterizing the 2-G PZT intra-cochlear actuator probe is the same as that for the 1-G probe (cf. Figure16 of [27]). Namely, the bottom electrode is first grounded. Then a 1-V swept sine signal from $10 \mathrm{~Hz}$ to $102.4 \mathrm{kHz}$ is generated via a spectrum analyzer (Stanford Research Systems, Model SR785). The signal is then amplified via a power amplifier (AVC Instrumentation, 790 Series) to drive the top electrode (i.e., the PZT thin-film micro-actuator diaphragms). In the meantime, a laser Doppler vibrometer (LDV, Polytec OFV511 \& 3001) measures the velocity of the center of the top electrode. The LDV measurement and the driving voltage are fed back to the spectrum analyzer to obtain a frequency response function (FRF).

\subsection{Measured Response and Discussions}

Figure 10 shows the measured FRF for the first diaphragm (i.e., the one closest to the tip; see Figure 8). In particular, the FRF curve in the low frequency range is flat with a constant gain of $0.44 \mathrm{~nm} / \mathrm{V}$ (i.e., $-7.1 \mathrm{~dB}$ ), and the first natural frequency is $60.06 \mathrm{kHz}$. For the partially released diaphragm simulated in Figure 3, however, the static gain is $12.62 \mathrm{~nm} / \mathrm{V}$ and the first natural frequency is $86.64 \mathrm{kHz}$. Obviously, the measured results are far different from the predicted results. Moreover, there is one very subtle self-contradictory paradox. On one hand, the measured natural frequency is lower than predicted implying that the partially released diaphragm is softer than designed. At the same time, the measured gain is also lower than predicted implying that the partially released diaphragm is stiffer than designed. The measured results seem to contradict each other. What is a proper explanation of these results? What is the actual physics behind the measured results? 


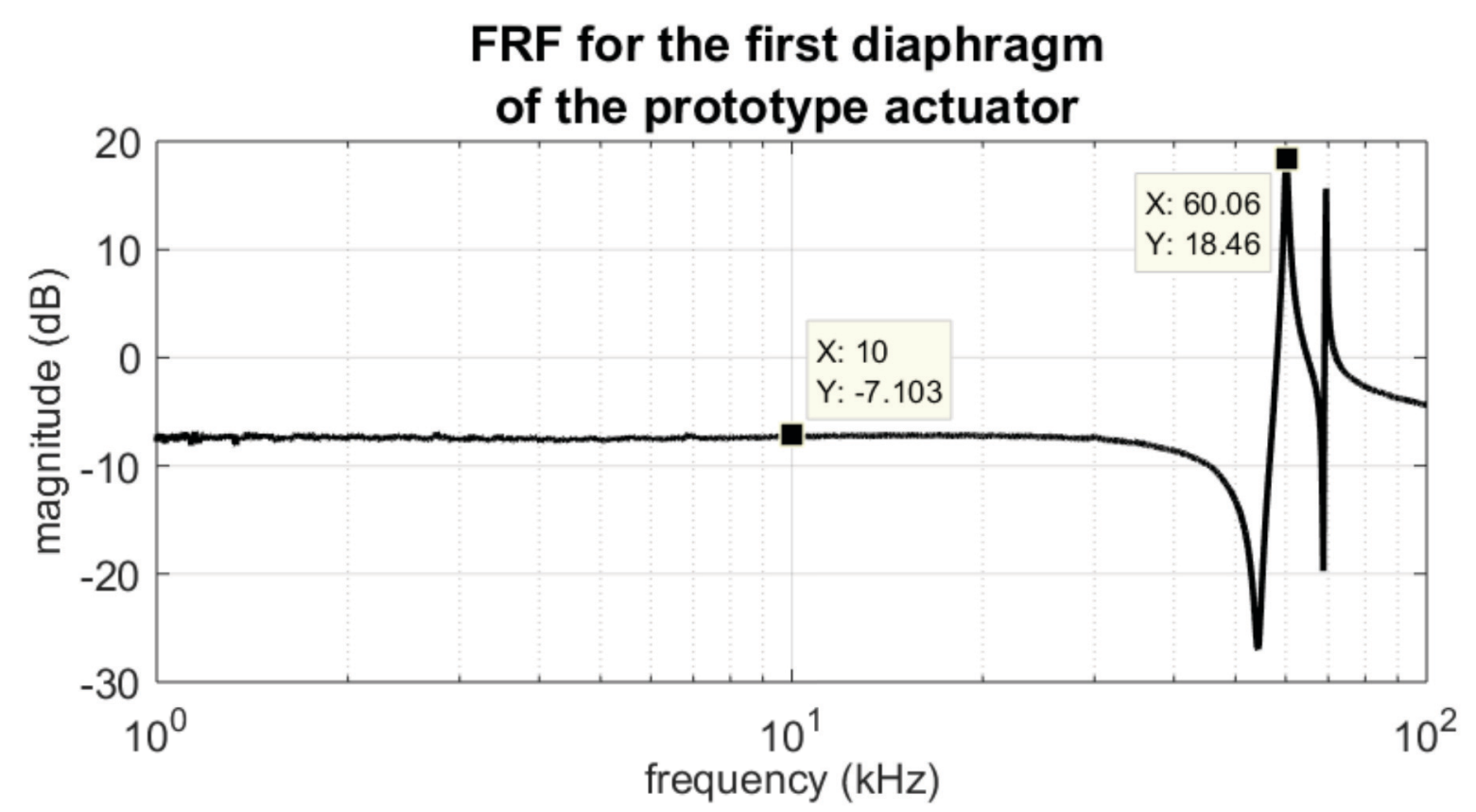

Figure 10. Frequency response function of the first partially released PZT diaphragm

For the rest of the section, we use the following three statements to uncover the physics behind the measured results. These statements are supported by rigorous experimental evidence, and they are explained in detail as follows.

Statement 1. The natural frequency at $60.06 \mathrm{kHz}$ is from the cantilever vibration of the $2-G$ probe; it is not from the resonance of the partially released diaphragms.

To support the statement, we use another 2-G probe to conduct the following experiment. First, an FRF of the 2-G probe is measured using the boundary conditions shown in Figure 9(a). Then, the boundary condition is changed; more epoxy is applied to the dashed square area highlighted in Figure 9(b), thus reducing the cantilever length of the 2-G probe. The FRF of the 2-G probe is measured again. The rationale of the experiment is the following. If the first 
natural frequency in the FRF results from the diaphragm resonance, the presence of the additional epoxy will not affect the first natural frequency. In contrast, if the first natural frequency results from the cantilever vibration, the presence of the additional epoxy will reduce the length of the cantilever and thus significantly increases the first natural frequency. Figure 11(a) and (b) compare the FRF before and after the excessive epoxy is applied. The first natural frequency increases from $50.07 \mathrm{kHz}$ to $88.07 \mathrm{kHz}^{2}$. Therefore, the first natural frequency results from a cantilever bending mode of the $2-\mathrm{G}$ micro-actuator probe.

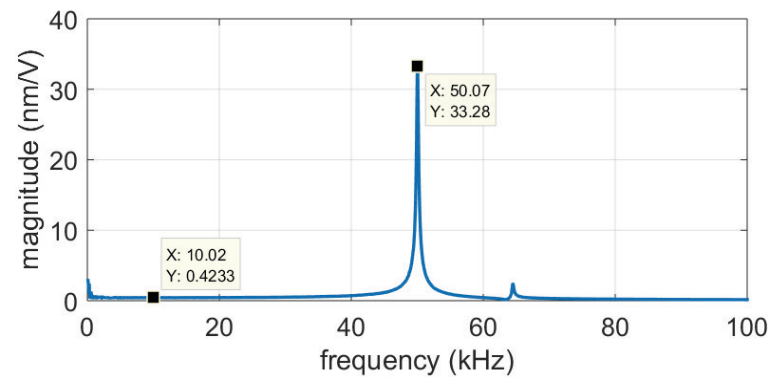

(a) FRF of the 2-G probe without excessive epoxy; first natural frequency $=50.07 \mathrm{kHz}$

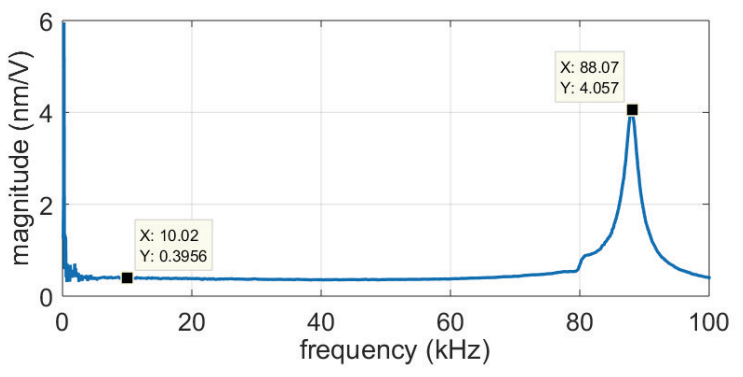

(b) FRF of the 2-G probe with excessive epoxy; first natural frequency $=88.07 \mathrm{kHz}$

${ }^{2}$ Recall that the probes resulting in Figures 10 and 11 are two different probes. 


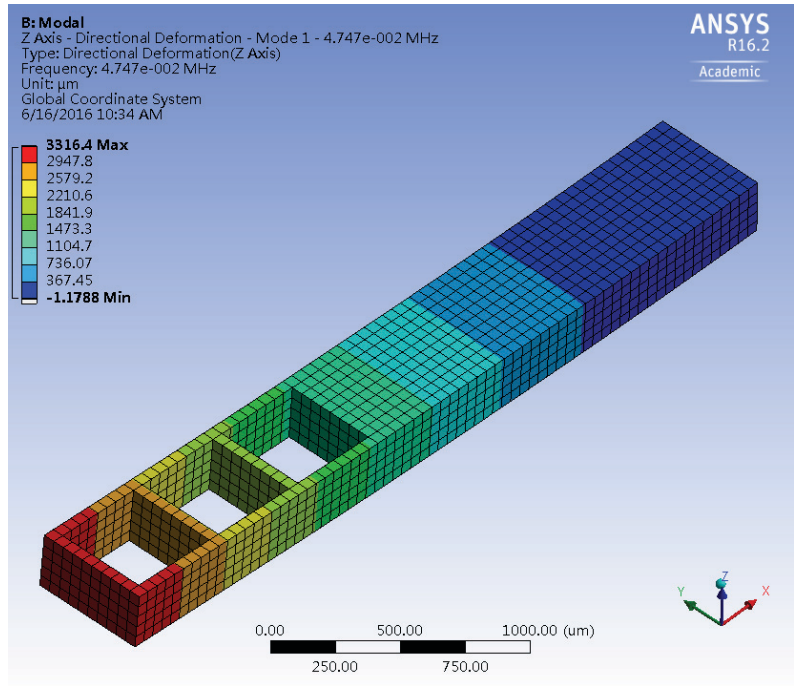

(c) modal analysis of the probe without excessive epoxy, first natural frequency $=47.47 \mathrm{kHz}$

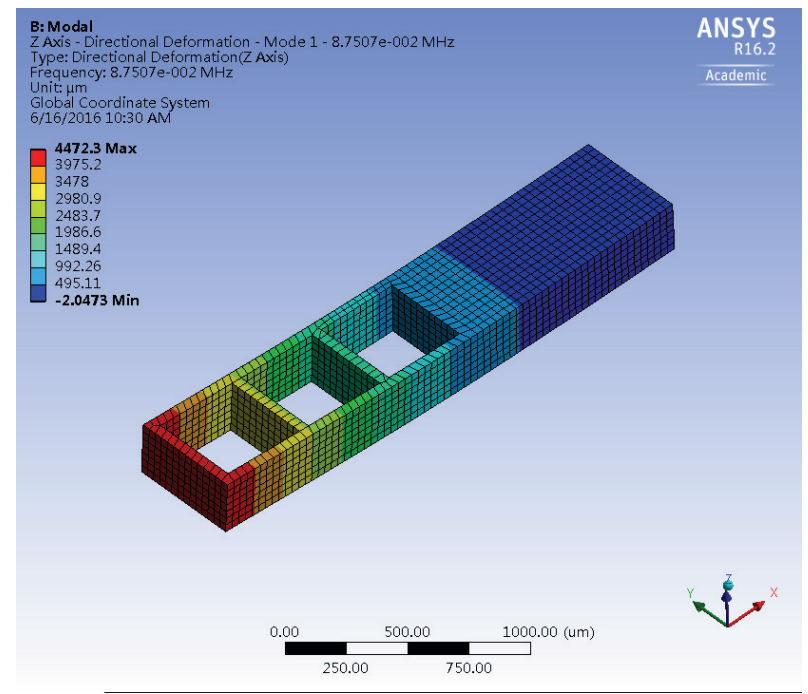

(d) modal analysis of the probe with excessive epoxy, first natural frequency $=87.51 \mathrm{kHz}$

Figure 11. FRFs and finite element models of the 2-G probe before and after excessive epoxy is applied: (a) FRF of the probe without excessive epoxy, (b) FRF of the probe with excessive epoxy, (c) modal analysis of the probe without excessive epoxy, (d) modal analysis of the probe with excessive epoxy

A finite element analysis is also conducted to confirm the cantilever vibration mode as the first resonance. The length of the $2-\mathrm{G}$ probe is measured roughly as $3500 \mu \mathrm{m}$ and $2500 \mu \mathrm{m}$, respectively, before and after the excessive epoxy is applied. Since the diaphragms have virtually no effect on the bending rigidity of the $2-\mathrm{G}$ probe, they are not included in the finite element model. A modal analysis is performed and the results are shown in Figure 11(c) and (d). The first vibration mode is cantilever bending. The corresponding natural frequency is $47.47 \mathrm{kHz}$ and $87.51 \mathrm{kHz}$ before and after the excessive epoxy is applied, respectively. The predicted natural frequencies agree well with the experimental measurements within 5\% (cf. $50.07 \mathrm{kHz}$ and 88.07 
$\mathrm{kHz}$ ). Therefore, the first mode measured by LDV is in fact the cantilever bending instead of the diaphragm resonance.

Statement 2. The constant gain $(0.44 \mathrm{~nm} / \mathrm{V})$ results from the deflection of the diaphragm and not from the cantilever probe deflection.

To support this statement, we measure the FRF at two different points. One is at the center of the third diaphragm (i.e., point A of Figure 8) and the other is at the outer electrode near the bulk silicon (i.e., point B of Figure 8). The rationale is the following. If the static gain results from the diaphragm deformation, the displacement (and hence the FRF) measured at points A will be substantially larger than that measured at point B. In contrast, if the static gain results from the cantilever beam bending, the displacement (and hence the FRF) measured at points A will be about the same as that measured at point B. The measurements are shown in Figure 12, with the blue and the red curves representing FRF of points A and B, respectively. In particular, FRF shows a static gain of $-7.732 \mathrm{~dB}(0.41 \mathrm{~nm} / \mathrm{V})$ at point $A$ as opposed to $-29.33 \mathrm{~dB}(0.034 \mathrm{~nm} /$ V) at point B. The drastic difference in the measurements confirms that the static gain results from the diaphragm deflection. 


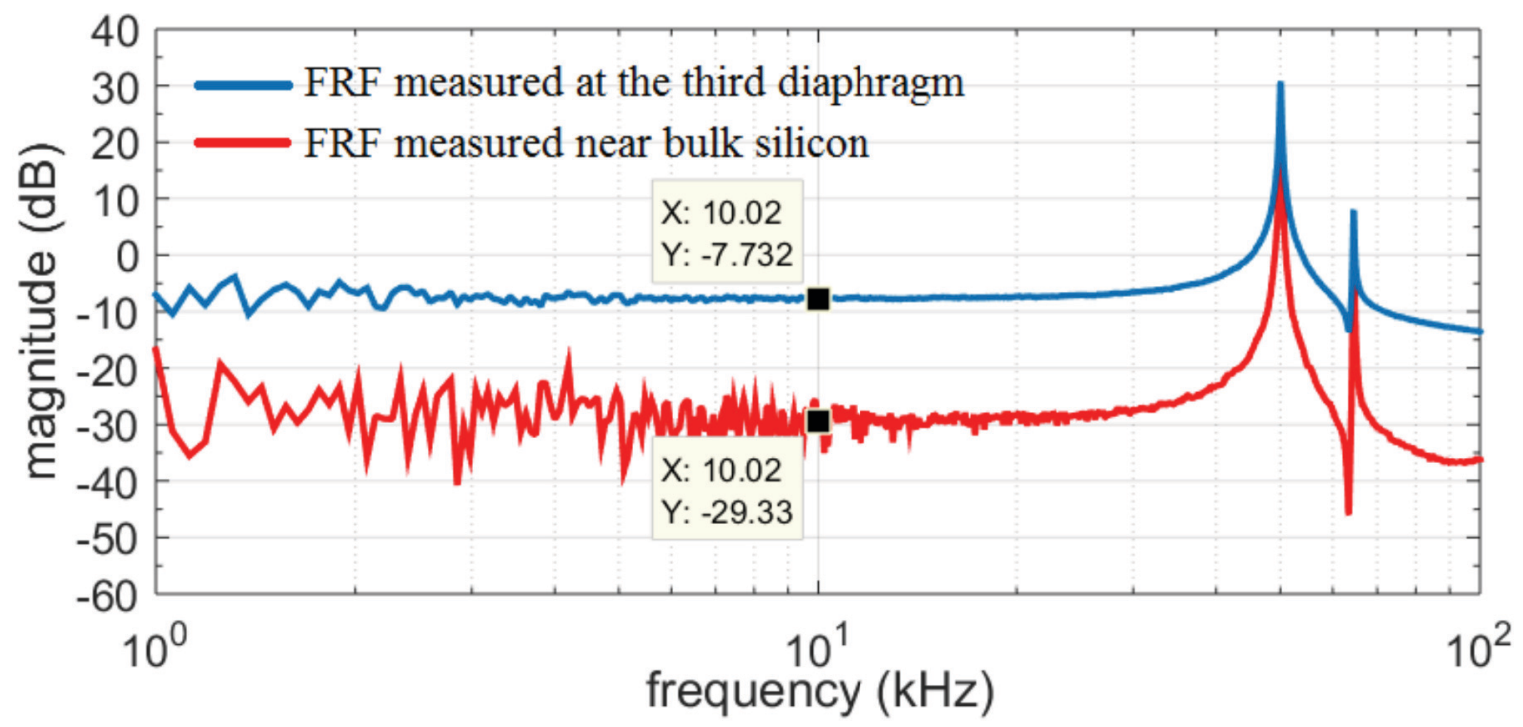

Figure 12. The frequency response functions of the actuator measured at the third diaphragm: at the center and at the outer electrode near bulk silicon

Statement 3. The low constant gain of $0.44 \mathrm{~nm} / \mathrm{V}$ in comparison with the designed gain $12.62 \mathrm{~nm} / \mathrm{V}$ results from under-etching of the diaphragm.

Figure 13(a) shows the bottom view of a properly etched diaphragm and an over-etched diaphragm under an optical microscope. For the properly etched diaphragm, the elliptical area in blue and pink is the diaphragm consisting of silicon nitride and oxide layers. The black and white area outside the ellipse is the residual silicon. The size of the residual silicon is $130 \mu \mathrm{m}$ in the longitudinal direction and $110 \mu \mathrm{m}$ in the transverse direction. Although the geometry and layout are identical to those depicted in the finite element model Figure 3(b), the residual silicon occupies a much larger area. Moreover, the over-etched diaphragm fractured and was not functional. Figure 13(b), in contrast, shows the bottom view of two partially released diaphragms of the 2-G probe yielding $0.44 \mathrm{~nm} / \mathrm{V}$ in Figure 10. Obviously, the diaphragm is under-etched, and 
un-etched silicon spreads over the entire diaphragm. The thickness of the un-etched silicon, estimated from the average etching rate of DRIE, is roughly $4-8 \mu \mathrm{m}$.

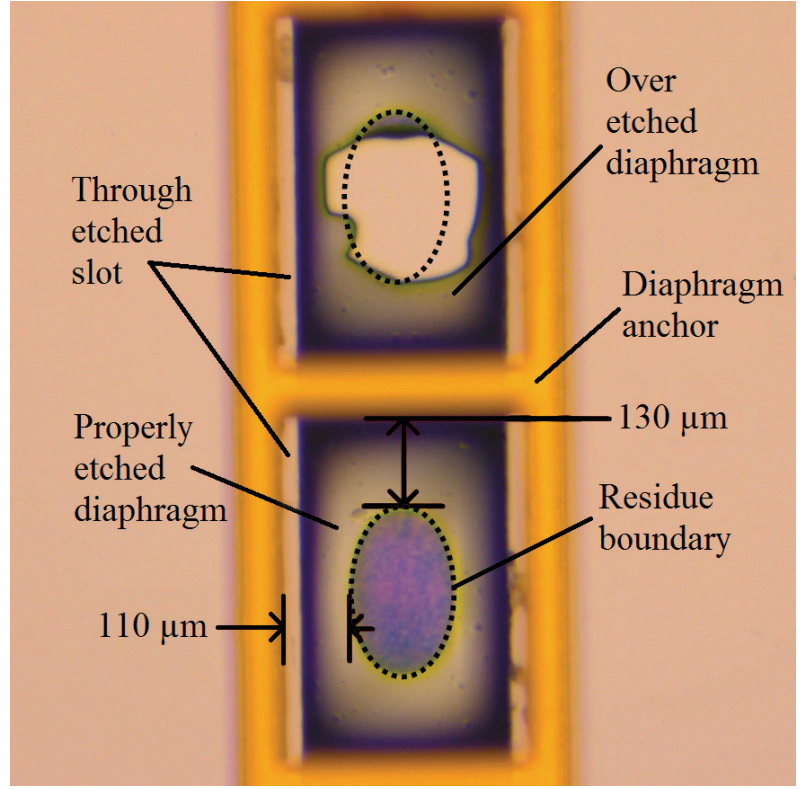

(a) Bottom view of a properly etched diaphragm and an over-etched diaphragm

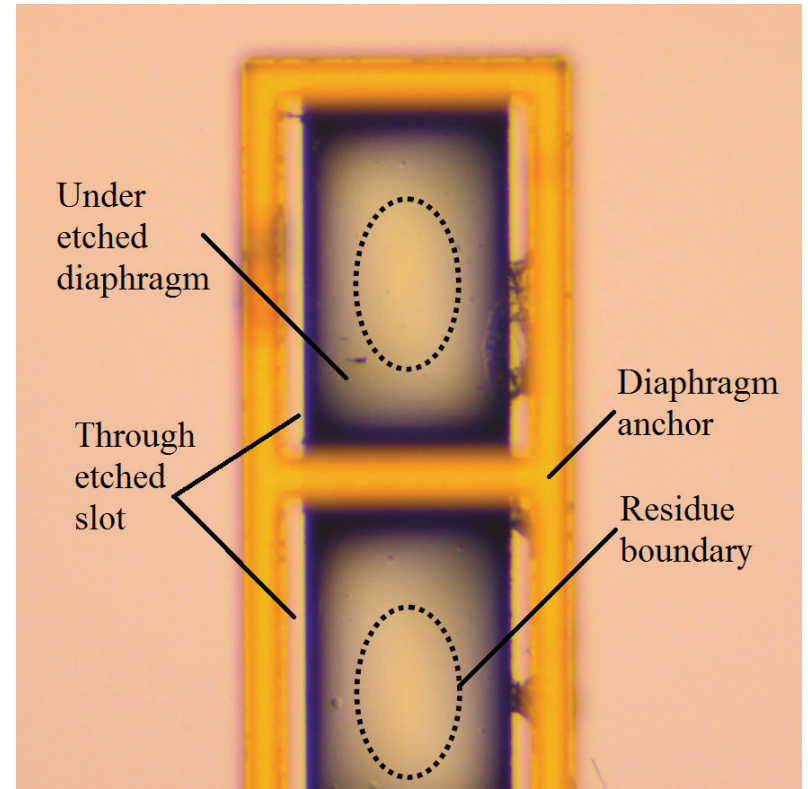

(b) Bottom view of two under-etched diaphragms

Figure 13. Bottom view of the 2-G micro-actuator probe indicating: (a) a properly etched diaphragm and an over-etched diaphragm, (b) two under-etched diaphragms

To evaluate the influences of the under-etched condition in Figure 13(b), we assume that the residual silicon has a longitudinal dimension of $130 \mu \mathrm{m}$ and a transverse dimension of 110 $\mu \mathrm{m}$. We further assume that the un-etched silicon layer is $4 \mu \mathrm{m}$. Based on these dimensions, we repeat the finite element analysis shown in Figure 3. The static gain of the diaphragm is substantially reduced from $12.62 \mathrm{~nm} / \mathrm{V}$ to $1.31 \mathrm{~nm} / \mathrm{V}$, while the natural frequency increases from $86.64 \mathrm{kHz}$ to more than $600 \mathrm{kHz}$. Note that the natural frequency did not appear in FRF 
measurements (cf. Figures 10 and 12), because it is out of the 102.4-kHz bandwidth of the spectrum analyzer. The finite element simulation indicates that the final dimensions and geometry of the un-etched silicon are very critical; they can significantly affect the static gain and the natural frequency of the partially released PZT thin-film microactuator.

Since we do not know the exact dimensions of the partially released diaphragm, we cannot match the finite element predictions with the experimental measurements. Nevertheless, it is well known that finite element analyses predict natural frequencies and static gains quite accurately for a simple structure, provided that accurate geometry and material properties are available. A good example is the frequency prediction of the cantilever shown in Figure 11. We are able to predict the measured natural frequencies within 5\%. Therefore, the finite element predictions in this paper should be fairly reliable and can be realized experimentally if the partially released microactuator can be fabricated to the designed dimensions.

In summary, the case study of 2-G intra-cochlear micro-actuator probes leads to the following major results. First, a partially released diaphragm is feasible for a micro-actuator, and it is theoretically feasible for a micro-sensor. We demonstrated the feasibility by fabricating one for intra-cochlear applications. Second, supporting structures of the partially released diaphragm (e.g., a cantilever) may affect the designed performance. For example, the first natural frequency may be controlled by the supporting structure of the 2-G micro-actuator probe instead of the diaphragm resonance. Finally, the static gain results from the deflection of the diaphragm, and it is largely controlled by the amount of un-etched silicon. 
In light of the summary above, there are two additional issues worth discussing. First, the three statements and major results above are direct consequences of the structural design. Therefore, the two probes shown in Figures $7(a)$ and $7(b)$ should have the same structural behavior, although the probe in Figure 7(b) is never fabricated. Second, the measured cantilever mode is not likely to matter in viable intra-cochlear applications. As described earlier, the cantilever structure was used to facilitate acute in-vivo tests. Even in those cases, the cantilever mode will not matter as long as it is outside the frequency range of the tests (e.g., $24 \mathrm{kHz}$ in [21]). For chronic in-vivo tests or viable hearing devices, the micro-actuator must be completely implanted. The cantilever length, if there is one, will be extremely small. Therefore, the cantilever mode will have extremely high natural frequency, and becomes totally irrelevant.

\section{FINAL REMARKS}

From the study above, it is certainly feasible to design, fabricate, and instrument a piezoelectric thin-film micro-actuator with partially released diaphragms. Naturally, a lingering question is whether or not a partially released diaphragm micro-actuator with a reduced size could outperform a fully anchored diaphragm micro-actuator. If yes, what could be the geometry and dimensions of such partially released diaphragm micro-actuators? The easiest way to answer is to conduct a parametric study and identify some favorable parameter combinations.

The parametric study leads to two major results. First, the thickness of the un-etched silicon layer needs to be precisely controlled. Figure 14 shows how sensitive the deflection of a partially released diaphragm varies with respect to the thickness of the un-etched silicon layer. 
The deflection peaks when the thickness is around $0.4 \mu \mathrm{m}$ and diminishes dramatically there from. This phenomenon occurs because the PZT thin film is very close to the neutral surface of the diaphragm. A tiny increase in the thickness of the un-etched silicon moves the PZT thin film away from the neutral surface, thus enhancing the diaphragm deflection. Too big an increase in the thickness, however, will significantly increase the flexural rigidity, thus reducing the deflection of the diaphragm.

The second major result is that complete removal of the residual silicon is a critical requirement to maximize the diaphragm deflection. If the through-etched slots do not remove enough residual silicon, the partially released diaphragm will have considerable flexural rigidity from the residual silicon limiting the diaphragm deflection. To maximize diaphragm deflection, it might be necessary to adopt larger through-etched slots.

These two major results, however, pose significant challenges for the fabrication of the microactuators. First of all, it is extremely difficult to etch the silicon to the desired thickness. As one can see in Figure 14, the static gain is very sensitive to the un-etched silicon. There is only a very tiny window (about $0.5 ? ? \mathrm{~m}$ ) to achieve a good result. This is a very challenging task in fabrication. Second, it is also very difficult to estimate and to control the size of the residual silicon. Therefore, it becomes extremely hard to determine how wide the through-etch slots should be before the fabrication starts. On the other hand, a mask must be made with welldefined through-etched slots before the etching begins. Due to these two challenges, we expect that a series of trials and errors must be performed in order to fabricate the microactuator to the desired dimensions for optimal performance. 
Figure 15 shows an actuator design that has a reduced size but significantly better static gain compared with the fully anchored diaphragm micro-actuator shown in Figure 16 of Appendix 1. The micro-actuator has a width of $700 \mu \mathrm{m}$ and a length of $750 \mu \mathrm{m}$ with a partially released diaphragm. This is equivalent to roughly a $50 \%$ reduction in total actuator area. In this design, the length and width of the through-etched slots is $750 \mu \mathrm{m}$ and $100 \mu \mathrm{m}$, respectively. Moreover, the thickness of the un-etched silicon layer is $0.7 \mu \mathrm{m}$. A finite element analysis indicates that the static deflection of the diaphragm is $29.59 \mathrm{~nm} / \mathrm{V}$ and the first natural frequency is $32.24 \mathrm{kHz}$. These numbers are comparable with $18.22 \mathrm{~nm} / \mathrm{V}$ and $35.51 \mathrm{kHz}$ of the fully anchored diaphragm micro-actuator shown in Figure 16. Note that the through-etched slots have completely removed the residual silicon on the backside of the diaphragm.

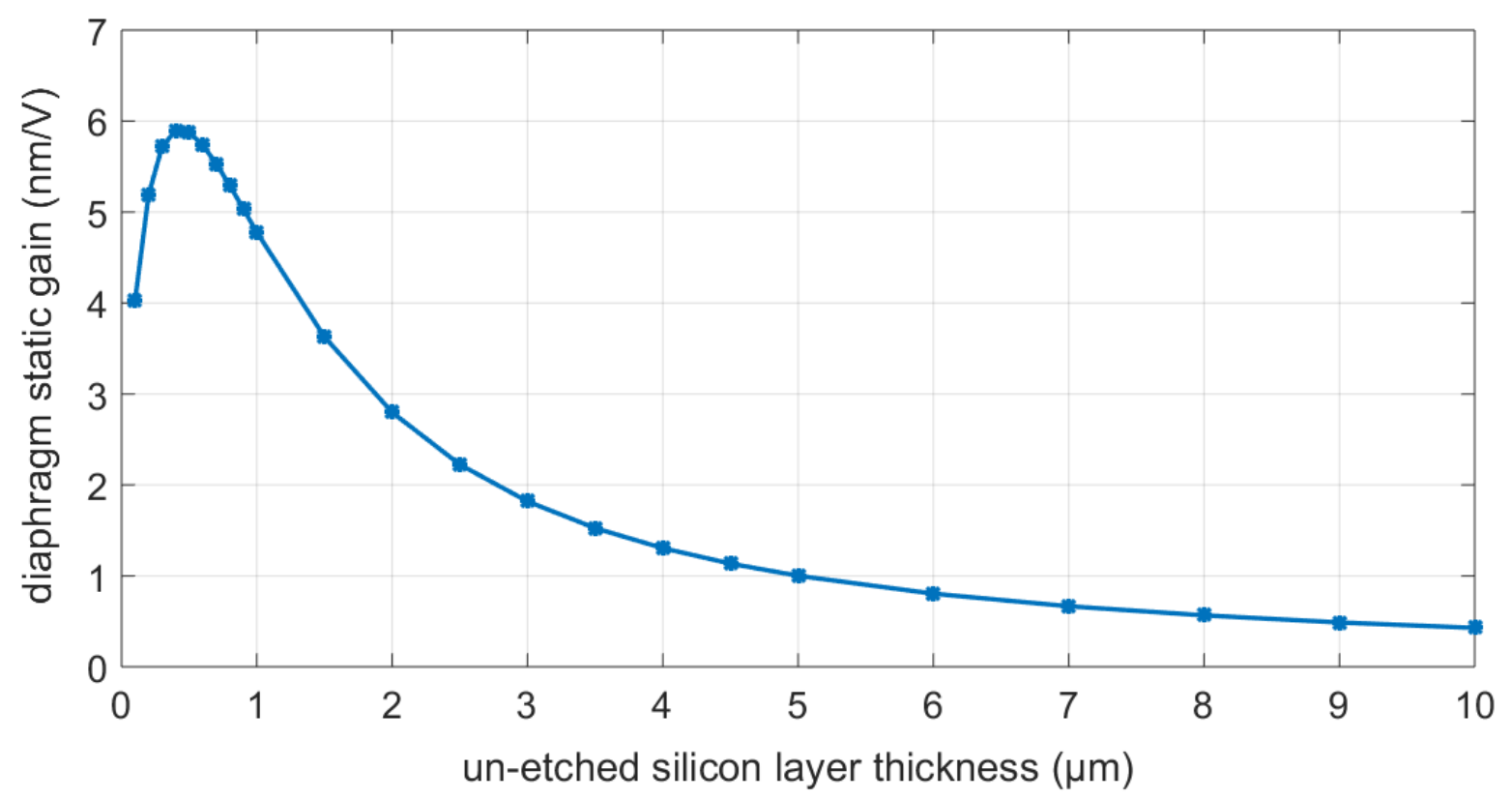


Figure 14. Parametric study of the un-etched silicon layer thickness with respect to the diaphragm static gain

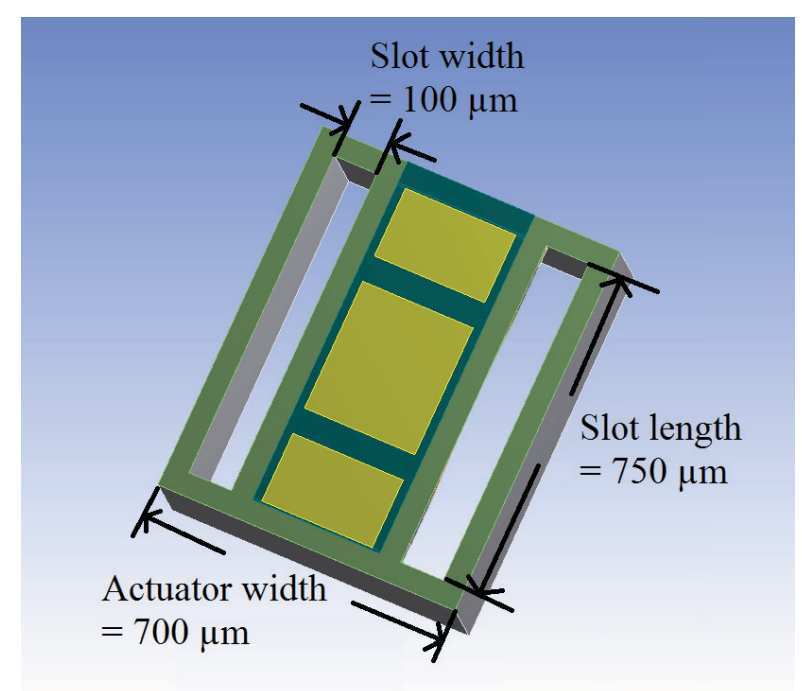

(a)front side of the optimized partially released actuator model

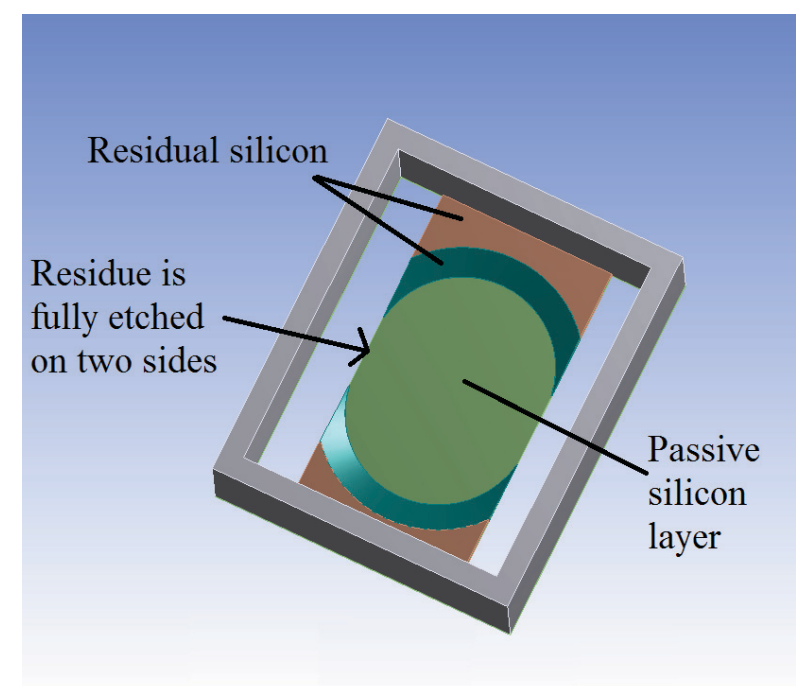

(b)back side of the optimized partially released actuator model

Figure 15. A partially released PZT micro-actuator after optimization:

(a) top view,(b) bottom view

\section{CONCLUSIONS}

Through the analyses, fabrication, instrumentation, tests, and discussions in this paper, we reach the following conclusions.

1. We proposed a partially released diaphragm micro-actuator that would considerably reduce the size of a fully anchored diaphragm micro-actuator while maintaining its static gain and bandwidth. We first confirm the feasibility of the design via rigorous finite 
element analyses. The finite element analyses indicate that tight control of the thickness of the un-etched silicon layer and complete removal of the residual silicon are two critical factors in reaching the desired performance.

2. New fabrication processes are developed to realize the through-etched slots that enable the partially released diaphragm design. Major fabrication challenges, such as presence of cat ear and inhomogeneity of bottom electrodes, are overcome.

3. To demonstrate the new design and fabrication process, a prototype is made for a secondgeneration, intra-cochlear, micro-actuator that houses three partially released diaphragms on a silicon cantilever with a cross section of $250 \mu \mathrm{m} \quad 500 \mu \mathrm{m}$. Experimental measurements indicate that the static gain results from the diaphragm deflection but the first natural frequency results from cantilever bending vibration. It is evident that the supporting structure of the partially released diaphragm (e.g., a cantilever) may affect the designed performance. Also, the test results confirm that control of the thickness of the un-etched silicon layer and complete removal of the residual silicon are critical in achieving the designed performance.

\section{ACKNOWLEDGMENT}

This material is based upon work supported by the National Science Foundation under Grant No. CBET-1159623. Any opinions, findings, and conclusions or recommendations expressed in this material are those of the authors and do not necessarily reflect the views of the 
National Science Foundation. The second author also thanks National Natural Science Foundation of China for its support through Grant No. 51405260. 


\section{APPENDIX 1: ANALYSIS OF FULLY ANCHORED DIAPHRAGM MICRO- ACTUATORS}

Figure 16 shows a finite element model of a fully anchored PZT thin-film diaphragm for the 1-G intra-cochlear micro-actuator probe. The actuator occupies a square domain $1 \mathrm{~mm} 1 \mathrm{~mm}$ with a thickness of $0.4 \mathrm{~mm}$; see Figure 16(a). In addition, the top surface has two gold electrodes: one central and one outer. Figure 16(b) shows the bottom view, which reveals a circular thin diaphragm, residual silicon, and diaphragm anchor. Note that the residual silicon consists of two parts. One is fully developed residual silicon that appears to be flat, and the other is a transition zone from the diaphragm to the fully developed residual silicon; see the schematic drawing in Figure 17 for detail. (Basically, Figure 17 is a schematic cross sectional view of Figure 16(b) at section A-A' but not in scale.) In Figure 17, Part A refers to the circular thin diaphragm, Part B is the transition zone, and part C is the fully developed (i.e., flat) residual silicon. Therefore, the blue and peach regions in Figures 16(b) are the transition zone and the fully developed residual silicon, respectively. The diameter of the circular diaphragm is $650 \mu \mathrm{m}$ and the wall thickness of the silicon anchors is $100 \mu \mathrm{m}$. Piezoelectric material properties are assigned based on previous studies in [31].

Figure 16(c) shows a cross-sectional view of the micro-actuator near an electrode ${ }^{3}$. The cross section of the diaphragm includes a top electrode $(\mathrm{Au})$, a PZT layer, a bottom electrode (Pt) and a passive silicon layer. (The passive silicon layer models silicon nitride, silicon oxide, and

\footnotetext{
${ }^{3}$ Since the aspect ratio (i.e., width-to-thickness ratio) of the cross section is very large (about 200:1), it is impossible to show the entire cross section with detail in a single plot. So we break it into two parts. The cross-sectional view near an electrode is shown in Figure 16(c), whereas the cross-sectional view near the residual silicon is shown schematically in Figure 17.
} 
un-etched silicon layers together.) Note that the diaphragm anchor shown in the cross-sectional view is not part of the diaphragm; it is only a projection of the anchor in the cross-sectional view. Also, the 1-G micro-actuator probe is fixed at one end; see Figure 16(a). Electric potential of 1V is applied to the top central electrode while the bottom electrode is grounded. The finite element simulations show that the static deflection of the circular diaphragm is $18.22 \mathrm{~nm} / \mathrm{V}$ and the first natural frequency is $35.51 \mathrm{kHz}$.

If the $1-G$ intra-cochlear micro-actuator is scaled down from $1 \mathrm{~mm}$ to $500 \mu \mathrm{m}$, the wall thickness of the silicon anchor is reduced to $50 \mu \mathrm{m}$. Since the residual silicon results from the non-uniform backside etch, its dimensions are not likely to change. As a result, the circular diaphragm will reduce to a diameter of $250 \mu \mathrm{m}$. Finite element simulations indicate that the static deflection of the diaphragm is only $3.39 \mathrm{~nm} / \mathrm{V}$. Also, the first natural frequency is 231.28 $\mathrm{kHz}$.

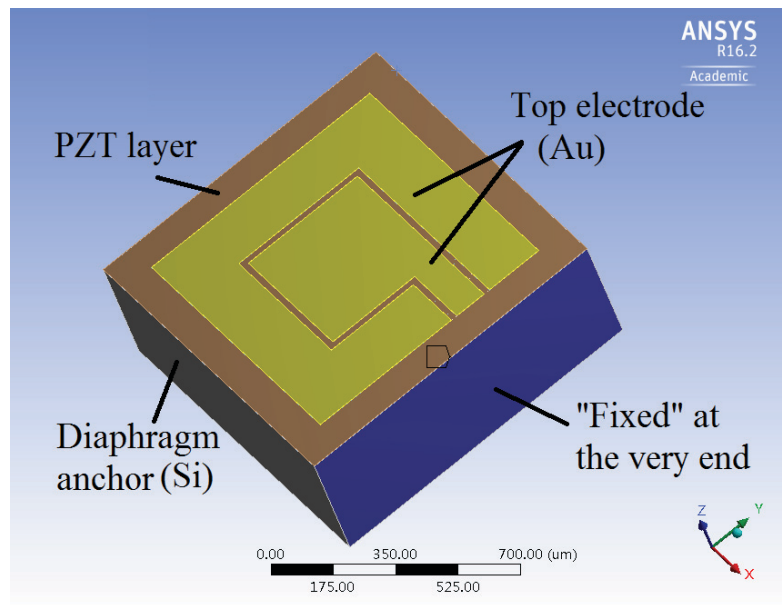

(a) front side view and boundary condition of the first-generation actuator model

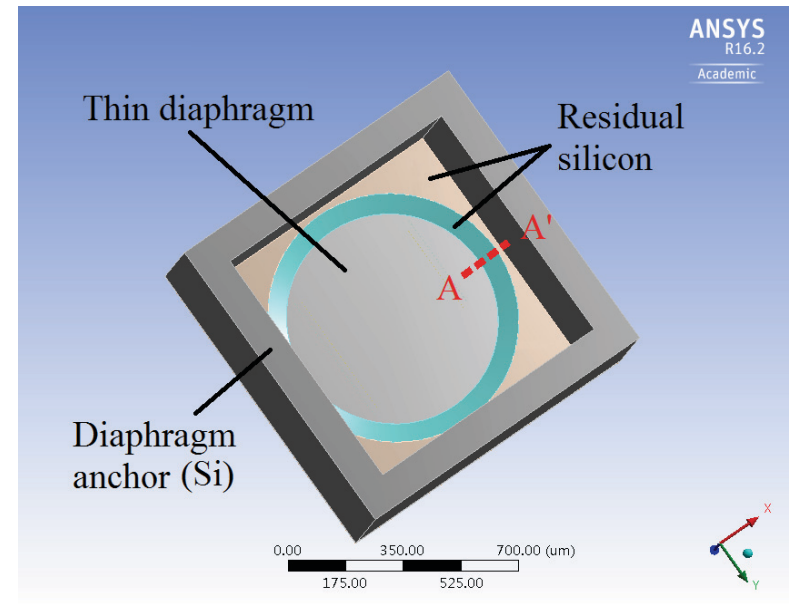

(b) back side view of the first-generation actuator model 


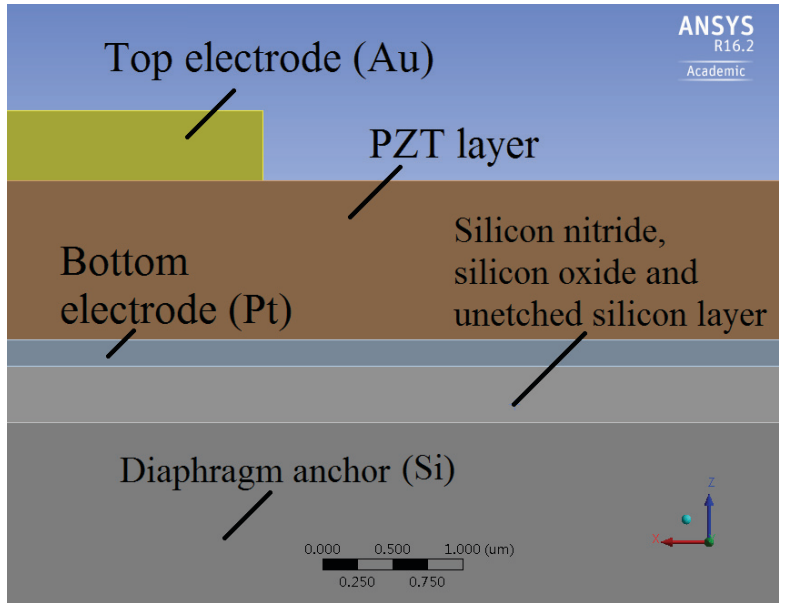

(c) Cross-sectional view of the actuator model near the top electrode

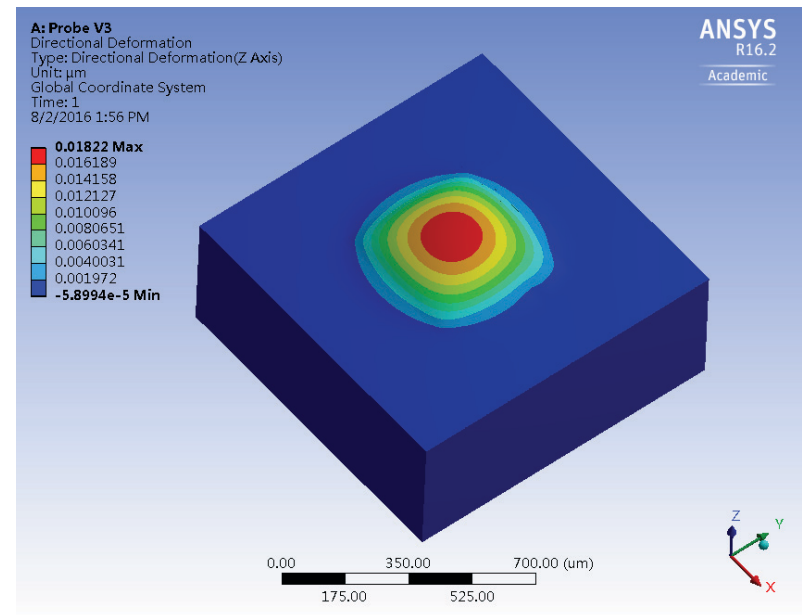

(d) diaphragm deflection from static analysis deflection $=18.22 \mathrm{~nm} / \mathrm{V}$

Figure 16. A fully anchored finite element model of the first-generation PZT micro-actuator:

(a) front side view and boundary condition, (b) back side view,

(c) cross-sectional view near an electrode, (d) static analysis.

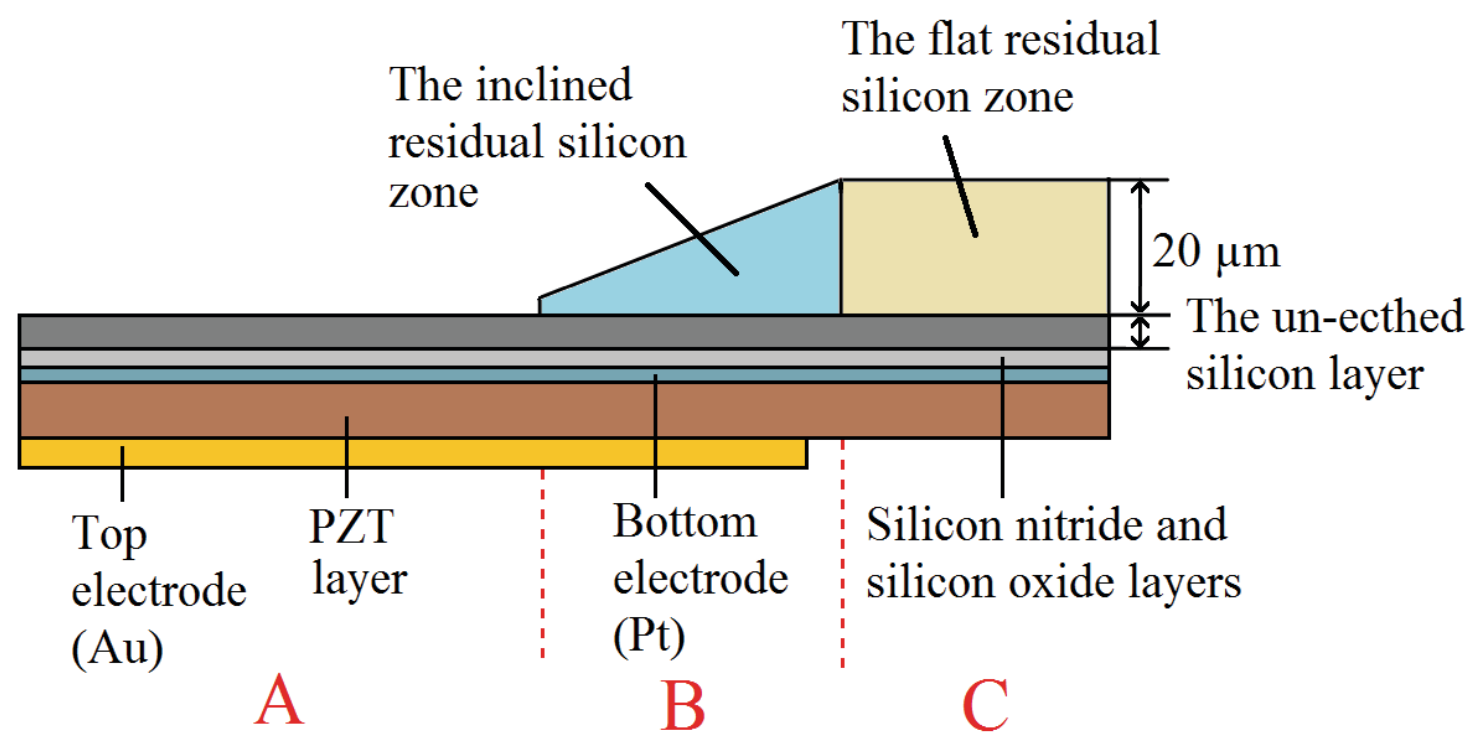

Figure 17.A schematic drawing of the cross-sectional view at the residual silicon (i.e. the cross section of A-A' in Figure 16(b) but not in scale) 


\section{REFERENCES}

1. E. Defay, C. Millon, C. Malhaire, and D. Barbier, "PZT thin films integration for the realization of a high sensitivity pressure micro-sensor based on a vibrating membrane," Sensors and Actuators A: Physical, vol. 99, no. 1, pp. 64-67, 2002.

2. M. Olfatnia, T. Xu, J. Miao, L. Ong, X. Jing, and L. Norford, "Piezoelectric circular microdiaphragm based pressure sensors," Sensors and Actuators A: Physical, vol. 163, no. 1, pp. 32-36, 2010 .

3. C. Luo, G. Cao, and I. Shen, "Development of a lead-zirconate-titanate (PZT) thin-film micro-actuator probe for intra-cochlear applications,"Sensors and Actuators A: Physical, vol. 201, pp. 1-9, 2013.

4. C. Luo, G. Cao, and I. Shen, "Enhancing displacement of lead-zirconate-titanate (PZT) thinfilm membrane micro-actuators via a dual electrode design," Sensors and Actuators A: Physical, vol. 173, no. 1, pp. 190-196, 2012.

5. C.-C. Lee, Q. Guo, G. Cao, and I. Shen, "Effect of electrode size and silicon residue on piezoelectric thin-film membrane actuators," Sensors and Actuators A: Physical, vol. 147, no. 1, pp. 279-285, 2008. 
6. T. Xu, Z. Wang, J. Miao, L. Yu, and C. M. Li, "Micro-machined piezoelectric membranebased immunosensor array," Biosensors and Bioelectronics, vol. 24, no. 4, pp. 638-643, 2008.

7. L. Nicu, M. Guirardel, F. Chambosse, P. Rougerie, S. Hinh, E. Trevisiol, J.-M. Francois, J.-P. Majoral, A.-M. Caminade, E. Cattan, and others, "Resonating piezoelectric membranes for microelectromechanically based bioassay: detection of streptavidin-gold nanoparticles interaction with biotinylated DNA," Sensors and Actuators B: chemical, vol. 110, no. 1, pp. 125-136, 2005.

8. T. Kawashima, Y. Yamada, Y. Matsuzawa, M. Nagai, T. Shibata, T. Masuzawa, T. Kimura, and A. Kishida, "Development of Cell Culture Microdevice Integrated with Piezoelectric Thin Film Actuator for On-Chip Regulation of Cell Functions," Japanese Journal of Applied Physics, vol. 51, no. 10R, p. 107201, 2012.

9. H. Ma, B. Hou, H. Wu, C. Lin, J. Gao, and M. Kou, "Development and application of a diaphragm micro-pump with piezoelectric device," Microsystem Technologies, vol. 14, no. 7, pp. 1001-1007, 2008.

10. N.-T. Nguyen and T.-Q. Truong, "A fully polymeric micropump with piezoelectric actuator," Sensors and Actuators B: Chemical, vol. 97, no. 1, pp. 137-143, 2004.

11. E. S. Kim and R. Muller, "IC-processed piezoelectric microphone," IEEE electron device letters, vol. 8, no. 10, pp. 467-468, 1987. 
12. E. Kim, J. Kim, and R. Muller, "Improved IC-compatible piezoelectric microphone and CMOS process," in Solid-State Sensors and Actuators, 1991. Digest of Technical Papers, TRANSDUCERS'91., 1991 International Conference on, 1991, pp. 270-273.

13. W. S. Lee and S. S. Lee, "Piezoelectric microphone built on circular diaphragm," Sensors and Actuators A: Physical, vol. 144, no. 2, pp. 367-373, 2008.

14. M. D. Williams, B. A. Griffin, T. N. Reagan, J. R. Underbrink, and M. Sheplak, "An AlN MEMS piezoelectric microphone for aeroacoustic applications," Journal of Microelectromechanical Systems, vol. 21, no. 2, pp. 270-283, 2012.

15. D. Kim, N. N. Hewa-Kasakarage, M. L. Kuntzman, K. D. Kirk, S. H. Yoon, and N. A. Hall, "Piezoelectric micromachined microphones with out-of-plane directivity," Applied Physics Letters, vol. 103, no. 1, p. 13502, 2013.

16. L. Baumgartel, A. Vafanejad, S.-J. Chen, and E. S. Kim, "Resonance-enhanced piezoelectric microphone array for broadband or prefiltered acoustic sensing," Journal of microelectromechanical systems, vol. 22, no. 1, pp. 107-114, 2013.

17. M. L. Kuntzman, N. N. Hewa-Kasakarage, A. Rocha, D. Kim, and N. A. Hall, "Micromachined In-Plane Pressure-Gradient Piezoelectric Microphones," IEEE Sensors Journal, vol. 15, no. 3, pp. 1347-1357, 2015.

18. B. Lee, S. Lin, W. Wu, X. Wang, P. Chang, and C. Lee, "Piezoelectric MEMS generators fabricated with an aerosol deposition PZT thin film," Journal of Micromechanics and Microengineering, vol. 19, no. 6, p. 65014, 2009. 
19. F. Lu, H. Lee, and S. Lim, "Modeling and analysis of micro piezoelectric power generators for micro-electromechanical-systems applications," Smart Materials and Structures, vol. 13, no. 1, p. $57,2003$.

20. E. Minazara, D. Vasic, F. Costa, and G. Poulin, "Piezoelectric diaphragm for vibration energy harvesting," Ultrasonics, vol. 44, pp. e699-e703, 2006.

21. C. Luo, I. Omelchenko, R. Manson, C. Robbins, E. C. Oesterle, G. Z. Cao, I. Shen, and C. R. Hume, "Direct Intra-cochlear Acoustic Stimulation Using a PZT Micro-actuator," Trends in hearing, vol. 19, p. $2331216515616942,2015$.

22. C. R. Knick and C. J. Morris, "Material and Process Development of Thin Film Shape Memory Alloy for MEMS Actuator," in ASME 2015 Conference on Smart Materials, Adaptive Structures and Intelligent Systems, 2015, p. V001T01A001-V001T01A001.

23. S. Watanabe, T. Fujiu, and T. Fujii, "PZT thin film actuator/sensor for atomic force microscope," in Applications of Ferroelectrics, 1996. ISAF'96., Proceedings of the Tenth IEEE International Symposium on, 1996, vol. 1, pp. 199-204.

24. G. De Simoni, G. Signore, M. Agostini, F. Beltram, and V. Piazza, “A surface-acoustic-wavebased cantilever bio-sensor," Biosensors and Bioelectronics, vol. 68, pp. 570-576, 2015.

25. S. Zhang, L. Lou, and C. Lee, "Piezoresistive silicon nanowire based nanoelectromechanical system cantilever air flow sensor," Applied Physics Letters, vol. 100, no. 2, p. 23111, 2012. 
26. C.-C. Lee, G. Cao, and I. Shen, "Effects of residual stresses on lead-zirconate-titanate (PZT) thin-film membrane micro-actuators," Sensors and Actuators A: Physical, vol. 159, no. 1, pp. $88-95,2010$

27. C. Luo, "PZT Thin-Film Micro Probe Device with Dual Top Electrodes," University of Washington, 2013.

28. L.-P. Wang, R. Wolf, Q. Zhou, S. Trolier-McKinstry, and R. Davis, "Wet-etch patterning of lead zirconatetitanate (PZT) thick films for microelectromechanical systems (MEMS) applications," in MRS Proceedings, 2000, vol. 657, p. EE5-39.

29. Y.-C. Hsu, C.-C. Wu, C.-C. Lee, G. Cao, and I. Shen, "Demonstration and characterization of PZT thin-film sensors and actuators for meso-and micro-structures," Sensors and Actuators A: Physical, vol. 116, no. 3, pp. 369-377, 2004.

30. Q. Guo, "Development of Thin-Film Based Microdevices and Process Enhancement for Making the Same," PhD Dissertation, University of Washington, 2012.

31. Q. Guo, G. Cao, and I. Shen, "Measurements of piezoelectric coefficient d33 of lead zirconatetitanate thin films using a mini force hammer," Journal of Vibration and Acoustics, vol. 135, no. 1, p. 11003, 2013. 